\title{
On Sovereign Credit Migration: A Study of Alternative Estimators and Rating Dynamics*
}

\author{
Ana-Maria Fuertes and Elena Kalotychou ${ }^{\dagger}$ \\ Cass Business School, City University London, 106 Bunhill Row, London EC1Y $8 T Z$
}

February, 2006

\begin{abstract}
This paper investigates the finite-sample behaviour of sovereign credit migration estimators and analyzes the properties of the rating process. Through bootstrap simulations, we compare a discrete multinomial estimator and two continuous hazard rate methods which differ in that one neglects time heterogeneity in the rating process whereas the other accounts for it. The study is based on Moody's ratings 1981-2004 for 72 industrialized and emerging economies. Hazard rate estimators yield more accurate default probabilities. The time homogeneity assumption leads to underestimating the default probability and greater migration risk is inferred upon relaxing it. There is evidence of duration dependence and downgrade momentum effects in the rating process. These findings have important implications for economic and regulatory capital allocation and for the pricing of credit sensitive instruments.
\end{abstract}

Keywords: Sovereign credit risk; Rating transitions; Markov chain; Time heterogeneity; Rating momentum; Duration dependence.

JEL Classification: C13; C41; G21; G28.

\footnotetext{
*We are grateful to Moody's Investors Services for providing the data. We appreciate the helpful comments of Chris Brooks, Jerry Coakley, Sema Dube, Frank Packer, Ron Smith, Sotiris Staikouras and participants at the 2005 FMA International Conference in Chicago, the 2005 Computational Statistics and Data Analysis Conference in Limassol, Cyprus, and seminar participants at the University of Essex, Department of Accounting, Finance and Management. We are responsible for any errors.

†Corresponding author: Tel: $+44(0) 207 \quad 040$ 8647; Fax: $+44(0) \quad 207040$ 8881; E-mail address: e.kalotychou@city.ac.uk.
} 


\section{Introduction}

Several recent sovereign defaults on foreign currency denominated debt — the 1994-1995 Mexican-peso crisis, the 1997 Asian currency crisis, the 1998 Russian ruble devaluation and the 2001 credit failure in Argentina have increased the financial institutions' concerns about their risk exposure to emerging markets. Credit migration analysis plays today a major role in risk management, valuation and international capital allocation. Financial institutions and regulators use ratings to feed VaR models (e.g. JP Morgan's Credit Metrics), to price risky loans and to determine concentration limits. Furthermore, the New Basel Accord (Basel II, 2001) permits banks to use internal ratings to set the regulatory capital against their credit exposure. Finally, credit ratings are key inputs to prominent models for the term structure of credit spreads (Jarrow et al., 1997) and the pricing of credit derivatives (Kijima and Komoribayashi, 1998). All these applications require a mapping of rating histories into transition probability estimates.

The amount of debt defaulted and the subsequent scale of losses in recent sovereign crises have by far exceeded those of corporate defaults. In this context, the application of extant risk management approaches to sovereigns warrants particular attention. Sovereign migration modeling is fraught with difficulties, mainly because of data limitations - the rating samples are relatively small both in the time-series and crosssection dimensions. The accurate estimation of migration probabilities requires enough transitions from each rating category which makes the task rather challenging for low credit-quality issuers (emerging markets) because, until very recently, ratings were mainly produced for industrialized countries. All these issues raise concerns about the reliability of sovereign rating migration measures. One alternative is to employ samples of corporate bond ratings which have a much longer history. This presumes that the sovereign credit migration process resembles that of corporates but the empirical evidence reveals significant discrepancies between them. Jackson and Perraudin (2000) find that, on average, sovereign credit spreads are substantially smaller than those of equally rated corporates. Cantor and Packer (1996) find that sovereign ratings exhibit more discrepancies across agencies than corporate ratings. Nickell et al. (2000) show that the transition probabilities of US industrials differ significantly from those of equally rated sovereigns.

The usual framework for modeling rating migration is a discrete time-homogeneous Markov process. This approach is relatively coarse in that it disregards the exact timing of rating changes and the duration in each rating. The discrete multinomial estimator, typically adopted by leading rating agencies (Carty and Fons, 1993 ) and in most of the academic literature (Bangia et al., 2002), produces zero probability for unobserved 
transitions by construction. For corporate ratings, Lando and Skodeberg (2002) illustrate how continuous hazard rate methods are able to provide non-zero probability for unobserved transitions (e.g. default from Aa). These risk measures are of consequence because such transitions can actually occur. Basel II implicitly supports this argument by setting a minimum probability of $0.03 \%$ for such rare events. Furthermore, a positive risk weight is assigned for sovereigns rated Aa to Baa which implies that Basel II recognizes the possibility of default from these ratings. On the other hand, the homogeneity assumption refers to the migration risk being constant over time which, although simplifies estimation, is clearly questionable. Time heterogeneity is a stylized fact of corporate rating migrations and it has been attributed either to momentum and duration effects or to business cycle dependence. Several studies address the hypothesis of rating drift or momentum in corporate ratings which implies that prior rating changes have predictive power for the direction of future changes. Downgrade momentum in corporate ratings is supported by Altman and Kao (1992), Carty and Fons (1993), Altman (1998), Kavvathas (2001), Bangia et al. (2002) and Lando and Skodeberg (2002). The duration effect refers to the relationship between the time spent in a given rating and the transition probability. Lando and Skodeberg (2002) and Kavvathas (2001) both confirm earlier findings by Carty and Fons (1993) on the presence of duration effects in corporate ratings but the evidence on the sign of the relationship is mixed. The impact of business cycles on default risk for speculative bonds has been documented in Wilson (1997), Nickell et al. (2000), Kavvathas (2001) and Bangia et al. (2002).

To our knowledge, only two studies in the literature investigate the estimation of sovereign credit migration risk and both consider discrete approaches. Wei (2003) proposes a multi-factor Markov chain model that accommodates time heterogeneity but recognizes the limitations of the model for sovereign debt due to the small samples available. Hu et al. (2002) use sovereign default data in conjunction with bond ratings to overcome the small-sample problem in a homogeneous ordered probit framework. Very little is known on the finite-sample properties of credit migration estimators in the context of sovereigns. There are two studies available that conduct a comparison of estimators (Jafry and Schuermann, 2004; Christensen et al., 2004 ) but their focus is on corporate debt. One goal of this paper is to contrast different estimators of sovereign rating transition risk. In this regard, the study has two motivations. First, the extensive evidence that sovereign and corporate credit ratings behave differently prompts the thought that a given estimator may have different properties in these two scenarios. For instance, there is consensus that corporate rating transition probabilities are time-varying and therefore hazard rate methods that allow for this heterogeneity 
are superior. However, this has not been established for sovereigns as yet. Second, given that sovereign ratings are subject to less frequent transitions than corporate ratings, continuous methods are expected to be more accurate and efficient than discrete ones. Another goal is to test for the presence of two types of non-Markovian effects in the rating histories known as momentum and duration dependence.

The analysis is based on a sample of Moody's credit ratings 1981-2004 for 72 industrialized and emerging economies. The paper compares a discrete multinomial estimator widely used in the literature and two continuous hazard rate estimators which differ in that one imposes the time-homogeneity assumption whereas the other relaxes it. A metric based on the transition matrix's spectral decomposition is used to gauge the overall rating migration risk implied by these estimators. A bootstrap simulation framework is adopted to compare the finite-sample bias and variance of the estimators. Particular attention is paid to two issues. One is to what extent continuous (versus discrete) estimation brings efficiency gains in small samples. The other is how important it is to account for time heterogeneity in the rating process.

The contribution of this study to the credit risk literature is threefold. First, it investigates the relative value of continuous-time estimators that use the full information of exact transition dates and rating durations. Second, it addresses the issue of whether assuming time homogeneity results in credit risk measures with relatively large small-sample bias and efficiency loss. Third, it provides the first tests for the presence of momentum and duration dependence in sovereign ratings, both of which suggest non-Markov effects or non-time homogeneity. For this purpose, we employ spectral analysis and panel logit models. We find that the continuous, hazard rate estimators produce more reliable default probability estimates than the discrete, multinomial estimator. The time-homogeneity assumption leads to too small (underestimated) sovereign default probabilities and larger migration risk is implied upon relaxing it. Furthermore, there are significant downgrade momentum and duration effects in the sovereign rating process, consistent with the extant evidence on corporate ratings. These findings have implications for risk management and capital allocation.

The paper is organised as follows. Section 2 outlines the Markov process and the three estimators. Section 3 presents the data. Section 4 discusses the bootstrap experiments for the comparison of estimators and the main findings. Section 5 investigates the properties of the rating process. A final section concludes. 


\section{Methodology}

The mainstream literature on credit migration rests on two premises. First, the future rating only depends on the current rating and is independent of the rating history (Markovian property). Second, the transition probabilities are constant over time (homogeneity property). The Markov process can be described as follows.

Let $i=1,2, \ldots, K$ denote the available credit ratings. Moody's bond rating system has seven coarse states (Aaa, Aa, A, Baa, Ba, B, C), twenty three finer states (Aaa1, Aaa2, Aaa3, Aa1,..., Ca) plus the default (D) state. We adopt the broader scale $(K=8)$ and so $i=1$ is the highest (Aaa) rating with $i=8$ denoting default. Let $P(s, t)$ be the $K \times K$ transition probability matrix driving the continuous Markov chain so that

$$
p_{i j}(s, t)=\operatorname{Pr}\left(\eta_{t}=j \mid \eta_{s}=i\right), s<t
$$

is the probability that a sovereign rated $i$ at time $s$ is at $j$ at time $t$. If the Markov chain is homogeneous, then the probabilities only depend on the transition horizon $(\Delta t=t-s)$ but not explicitly on time and thus there is a family of matrices, $P_{\Delta t}$, indexed by $\Delta t$. The transition matrix becomes $\left(P_{\Delta t}\right)^{n}$ for horizon $n \Delta t .{ }^{1}$ The transition matrix for a homogeneous Markov chain over a one-period (i.e. $\Delta t=1$ ) horizon is denoted $P=\left\{p_{i j}\right\}$ for $i, j=1, \ldots, K$ with entries $p_{i j} \geq 0$ and $\sum_{j=1}^{K} p_{i j}=1$ for all $i$. Default is treated as an absorbing state and so $p_{K K}=1$ (for $K=8$ ) by construction. The counterpart matrix for a heterogeneous Markov chain is denoted $P(s, t)$. The remainder of this section discusses three alternative estimators of transition matrices and the bootstrap framework adopted to approximate their sampling distribution.

\subsection{Discrete Multinomial (DM) Estimator}

The conventional approach to the estimation of $P$ discretizes the above Markov process and assumes time homogeneity. This leads to the discrete multinomial (DM) estimator based on annual migration frequencies. Transitions from a given state $i=1,2, \ldots, K$ to another state $j$ over a one-year horizon are assumed to follow a multinomial distribution with $K-1$ outcomes and associated probabilities $p_{i j}, i \neq j$. Let $N_{i}(t)$ denote the number of sovereigns that start year $t$ in state $i$ and $N_{i j}(t, t+1)$ the number of those that have migrated to $j$ by the start of year $t+1$. The annual migration frequency from $i$ to $j$ is calculated as $\frac{N_{i j}(t, t+\mathbf{1})}{N_{i}(t)}$.

Let us assume homogeneous, Markov chain dynamics for the rating process. Accordingly, the maximum

\footnotetext{
${ }^{1}$ See Norris (1997) for an in-depth discussion of Markov chains.
} 
likelihood estimator (MLE) of the one-year transition probability is

$$
\hat{p}_{i j}=\sum_{t=1}^{T} w_{i}(t) \frac{N_{i j}(t, t+1)}{N_{i}(t)}=\frac{\sum_{t=1}^{T} N_{i j}(t, t+1)}{\sum_{t=1}^{T} N_{i}(t)}
$$

where $T$ is the number of sample years and $w_{i}(t)=\frac{N_{i}(t)}{\sum_{t=1}^{T} N_{i}(t)}$ is the weight for the migration frequency on year $t$. Many studies use instead a coarser estimator, $\tilde{p}_{i j}=\frac{1}{T} \sum_{t} \frac{N_{i j}(t, t+1)}{N_{i}(t)}$, the sample average of the year-on-year migration frequencies (Bangia et al., 2002; Hu et al., 2002). The latter coincides with (2) when the number of sovereigns that start year $t$ at rating $i$ remains constant over the sample period, $N_{i}(t)=N_{i}$, meaning that the annual rating inflow is assumed equal to the outflow. Estimator (2) neglects information about within-year rating transitions and rating duration. In the context of sovereigns, where rating transitions are scarce, it is crucial to exploit the full information. This motivates the following estimators.

\subsection{Homogeneous Hazard Rate (HHR) Estimator}

Let the generator or intensity matrix of a continuous Markov chain at time $t$ be denoted by $\Lambda_{t}=\left\{\lambda_{i j}(t)\right\}$ for $i, j=1, \ldots, K$ where the off-diagonal transition intensity

$$
\lambda_{i j}(t) \equiv \lim _{\Delta t \rightarrow 0^{+}} \frac{p_{i j}\left(t \leq \tau_{i} \leq t+\Delta t \mid \tau_{i} \geq t\right)}{\Delta t} \geq 0
$$

is defined as the instantaneous (at time $\tau=t$ ) rate of transition from rating $i$ to rating $j$ conditional upon surviving in state $i$ up to time $t$ and $\lambda_{K j}(t)=0$ for all $j$. The diagonal element

$$
\lambda_{i i}(t) \equiv \lambda_{i}(t) \equiv-\sum_{j=\mathbf{1 , j \neq i}}^{K} \lambda_{i j}(t), i \neq j
$$

is the hazard rate function or the instantaneous rate of transition away from $i$ at time $t$, conditional upon being in state $i$ up to time $t$ (the minus sign denotes outflow from rating $i$ ). Thus the probability of leaving rating $i$ over an arbitrary time horizon $\Delta t$ is equal to $\lambda_{i}(t) \Delta t$. The transition intensity $\lambda_{i j}$ is driven by process $\tau_{i}$, the duration or survival time in rating $i$, which is subject to censoring or discontinuation at both ends of the sample - right (left) censoring means that the duration of each issuer in a given rating after the end (before the start) of the sample is unknown.

In order to estimate $\Lambda$ under the assumption of independent durations, the contribution to the overall transition probability for rating $i$ of each possible duration or, equivalently, transition is accounted for (i.e. from $i$ to $j, j \neq i$ ). The contributions of $N_{i j}$ migrations (or durations) from state $i$ to state $j$ occurring 
at times $t_{m}, m=1, \ldots, N_{i j}$, and of $N_{i c}$ censored durations (at rating $i$ ) occurring at $t_{m}, m=1, \ldots, N_{i c}$, are incorporated in the likelihood function for the transition from state $i$ as follows

$$
L_{i}=\left[\prod_{j=1, j \neq i}^{K} \prod_{m=1}^{N_{i j}} \lambda_{i j}(t) \exp \left(-\int_{0}^{t_{m}}\left\{\sum_{j=1, j \neq i}^{K} \lambda_{i j}(t)\right\} d u\right)\right] \prod_{m=1}^{N_{i c}} \exp \left(-\int_{0}^{t_{m}}\left\{\sum_{j=1, j \neq i}^{K} \lambda_{i j}(t)\right\} d u\right)
$$

If the intensities are homogenous, $\Lambda_{t}=\Lambda$, maximisation of the $\log$-likelihood $\log L_{i}$ with respect to $\lambda_{i j}$ gives the MLE as a simple closed-form solution for the transition intensities

$$
\hat{\lambda}_{i j}=\frac{N_{i j}(T)}{\sum_{m=1}^{N_{i}} t_{m}}
$$

where $N_{i j}(T)$ is the number of transitions from $i$ to $j,(i \neq j)$, within the window $[0, T]$ such that the total number of countries in rating $i$ within this period is $N_{i}(T)=N_{i j}(T)+N_{i c}(T)$. Estimator (5) can be rewritten as $\hat{\lambda}_{i j}=\frac{N_{i j}(T)}{\int_{0}^{T} Y_{i}(u) d u}$ where $Y_{i}(u)$ is the number of sovereigns rated $i$ at time $u$ and so the denominator gives the total time spent in state $i$ by all the sampled sovereigns. The homogeneous hazard rate (HHR) estimator of the transition probabilities over horizon $\Delta t$ is $^{2}$

$$
\hat{P}=\exp (\hat{\Lambda} \Delta t), \Delta t \geq 0
$$

where the matrix exponential is calculated as $\exp (\hat{\Lambda} \Delta t)=\sum_{k=0}^{\infty} \frac{(\hat{\Lambda} \Delta t)^{k}}{k !}$.

The continuous (hazard rate) estimator, equation (6), has several advantages over the discrete (multinomial) counterpart, equation (2). First, suppose that a country's rating evolution is Aaa $\longrightarrow$ Aa $\longrightarrow$ A within the horizon of interest (say $\Delta t=1$ year), then the transition to Aa contributes to the estimation of the transition probabilities from Aaa to Aa and from Aa to A through (5) but not through (2). Second, it accounts for the exact date when a sovereign receives a new rating and also for each rating duration. In the above example, the time spent in the intermediate state Aa is accounted for in the estimation of the transition intensity $\hat{\lambda}_{i j}$ for $i=A a$. Third, it readily accommodates right censoring stemming from rating withdrawals by using information for the obligors up to the withdrawal day. Thus obligors ending the year as withdrawn ratings will not be discarded as in the DM estimator. Fourth, it generally yields non-zero probabilities of rare transitions between states, even if they are not observed in the sample, as long as an indirect transition from one state to the other occurs. The DM estimator would yield zero for these unobserved transitions. For instance, suppose that no direct defaults from state Aa are observed but there are migrations $\mathrm{Aa} \longrightarrow \mathrm{B}$ and

\footnotetext{
${ }^{2}$ It is only under homogeneity that a simple mapping from transition intensities to transition probabilities exists. A detailed exposition of failure time analysis can be found in Kalbfleisch and Prentice (2002).
} 
from $\mathrm{B}$ to default. Then as long as the probability of migrating to $\mathrm{B}$ and the default probability from $\mathrm{B}$ are both non-zero, the continuous estimator yields a non-zero default probability for Aa. Finally, the transition intensities can be easily transformed into transition probabilities over any time horizon.

\subsection{Non-Homogeneous Hazard Rate (NHHR) Estimator}

The nonparametric approach of Aalen and Johansen (1978) extends the discrete estimator (2) to a continuoustime estimator that allows for heterogeneity in the underlying Markov process. Let $P(s, t)$ be the rating transition matrix over horizon $[s, t]$ with entries as defined in (1). Assuming $N$ transitions occur within $[s, t]$, this matrix can be consistently estimated by means of the Aalen-Johansen non-parametric product limit

$$
\hat{P}(s, t)=\prod_{m=1}^{N}\left[I+\Delta \hat{A}\left(T_{m}\right)\right]
$$

where the time point $T_{m} \in[s, t]$ denotes a transition occurrence, $N$ is the total number of transitions (i.e. the number of days where at least one transition occurs) and

$$
\Delta \hat{A}\left(T_{m}\right)=\left[\begin{array}{ccccc}
-\frac{\Delta N_{1}\left(T_{m}\right)}{Y_{1}\left(T_{m}\right)} & \frac{\Delta N_{12}\left(T_{m}\right)}{Y_{1}\left(T_{m}\right)} & \frac{\Delta N_{13}\left(T_{m}\right)}{Y_{1}\left(T_{m}\right)} & \ldots & \frac{\Delta N_{1 K}\left(T_{m}\right)}{Y_{1}\left(T_{m}\right)} \\
\frac{\Delta N_{21}\left(T_{m}\right)}{Y_{2}\left(T_{m}\right)} & -\frac{\Delta N_{2}\left(T_{m}\right)}{Y_{2}\left(T_{m}\right)} & \frac{\Delta N_{23}\left(T_{m}\right)}{Y_{2}\left(T_{m}\right)} & \ldots & \frac{\Delta N_{2} K}{Y_{2}\left(T_{m}\right)} \\
\vdots & \vdots & \ddots & & \vdots \\
\frac{\Delta N_{K-1,1}\left(T_{m}\right)}{Y_{K-1}\left(T_{m}\right)} & \frac{\Delta N_{K-1,2}\left(T_{m}\right)}{Y_{K-1}\left(T_{m}\right)} & \ldots & -\frac{\Delta N_{K-1}\left(T_{m}\right)}{Y_{K-1}\left(T_{m}\right)} & \frac{\Delta N_{K-1, K}\left(T_{m}\right)}{Y_{K-1}\left(T_{m}\right)} \\
0 & 0 & \cdots & \cdots & 0
\end{array}\right]
$$

where $\Delta N_{i j}\left(T_{m}\right)$ is the number of transitions from state $i$ to $j$ at time $T_{m}$. The diagonal entries $\Delta N_{i .}\left(T_{m}\right)$ are the number of transitions away from state $i$ at time $T_{m}$ so that each row sums to zero and the rows of $I+\Delta \hat{A}\left(T_{m}\right)$ sum to 1 . The number of sovereigns in state $i$ just before $T_{m}$ is denoted $Y_{l}\left(T_{m}\right)$. The off-diagonal entries $\left\{\Delta \hat{A}\left(T_{m}\right)\right\}_{i j}, i \neq j$, represent the fraction of sovereigns rated $i$ just before $T_{m}$ that migrated to rating $j$ at $T_{m}$. The Aalen-Johansen method can be cast as the DM estimator (2) extended to infinitely short-time intervals (i.e. to each point of time, $T_{m}$ ). Finally, a long-run average transition matrix can be obtained as

$$
\hat{P}=w_{0} \hat{P}\left(t_{0}, t_{1}\right)+w_{1} \hat{P}\left(t_{1}, t_{2}\right)+\ldots+w_{T-1} \hat{P}(T-1, T)
$$

where $\hat{P}\left(t_{k}, t_{k+1}\right), k=0, \ldots, T-1$ are particularizations of $(7)$ for sequential, non-overlapping intervals $\left(t_{k}, t_{k+1}\right)$ and $w_{k}$ is the proportion of rated countries at $t_{k}$. 


\subsection{Rating Mobility Measures}

One key aspect in the analysis of credit risk is the overall rating mobility or dynamics as opposed to stability

- these two attributes refer to the off-diagonal and diagonal probability mass of a transition matrix, respectively. Mispredicting the off-diagonal probabilities and especially, the default risk entails large economic costs. In the literature, this comparison has been mostly based on Euclidean distances (Israel et al., 2001; Bangia et al., 2000) and indices of mobility for Markov matrices based on eigenvalues and determinants (Shorrocks, 1978; Geweke et al. 1986). A pitfall of these mobility metrics is that they cannot provide a clear signal with matrices that have large concentration of diagonal mass, a feature which is typical of sovereign data. To circumvent this problem we employ Jafry and Schuermann's (2004) mobility estimator.

The dynamic part of the system or overall mobility matrix is given by $\tilde{P}=P-I$ where the identity matrix $I$ represents a stable (no migrations) system. The Jafry-Schuermann estimator is

$$
m(\hat{P}) \equiv \frac{1}{K} \sum_{i=1}^{K}\left\{\sqrt{e_{i}\left(\tilde{P}^{\prime} \tilde{P}\right)}\right\}
$$

where $e_{i}\left(\tilde{P}^{\prime} \tilde{P}\right)$ is the $i^{t h}$ eigenvalue of $\tilde{P}^{\prime} \tilde{P}$. Given the mobility matrices $\tilde{P}_{A}$ and $\tilde{P}_{B}$, the metric $\Delta m\left(\hat{P}_{A}, \hat{P}_{B}\right)=$ $m\left(\hat{P}_{A}\right)-m\left(\hat{P}_{B}\right)$ captures the concentration of off-diagonal probability mass. If $\tilde{P}_{A}$ and $\tilde{P}_{B}$ have identical diagonal values, the conventional differential metrics in the literature are simply zero irrespective of the offdiagonal entries but this perverse effect does not occur in $\Delta m\left(\hat{P}_{A}, \hat{P}_{B}\right)$. When the off-diagonal probability is diluted across a number of entries, then (10) is smaller than when it is concentrated in a few positions.

\subsection{Bootstrap Distribution of Rating Migration Estimators}

Testing hypotheses and constructing confidence intervals on rating migration risk requires the estimators' sampling distributions. In order to derive the variance-covariance of the transition matrix estimator (6), asymptotic theory is needed to obtain not only the variance-covariance of the intensity matrix $\hat{\Lambda}$ but also that of the matrix exponential $\exp (\hat{\Lambda} \Delta t)$. Unfortunately, there is no simple closed-form expression for these variance-covariance matrices. The asymptotic distribution of the mobility differential estimator $\Delta m\left(\hat{P}_{A}, \hat{P}_{B}\right)$ is also unknown. Moreover, the sovereign rating histories available have relatively few transitions and so relying on asymptotic theory may be inappropriate. To circumvent these problems, we deploy Christensen et al.'s (2004) bootstrap approach to approximate the estimators' sampling distributions as follows.

The discrete (DM) and continuous (HHR) estimators are compared through the following experiment. 
First, the observed ratings are used to estimate the intensity matrix and one-day transition probability matrix, $\Lambda$ and $P=\exp \left(\frac{1}{365} \Lambda\right)$, respectively. The probability estimates, $\left\{\hat{p}_{i j}\right\}$, are adopted as 'true' parameters of the bootstrap data generating process (DGP). More specifically, the rating histories of the various obligors are conceptualized as realizations of a continuous, homogeneous Markov process. We thus construct $R$ bootstrap samples, $\left\{B_{j}\right\}_{j=1}^{R}$, each of them containing the same number of sovereign histories, $N$, as the observed sample. ${ }^{3}$ Each sovereign's lifetime $h$ and initial rating $X_{0}$ are also as in the sample.

The bootstrap daily rating histories are constructed as follows. For a given sovereign, suppose that $X_{0}=$ Baa and that the estimates for the transition probability from rating Baa to Aaa, Aa, A, Baa, Ba, B, $\mathrm{C}$ and D are $\hat{p}_{41}, \hat{p}_{42}, \hat{p}_{43}, \hat{p}_{44}, \hat{p}_{45}, \hat{p}_{46}, \hat{p}_{47}$ and $\hat{p}_{48}$, respectively, with $\sum_{j=1, j \neq 4}^{8} \hat{p}_{4 j}=1$. Eight cumulative probability ranges are formed, one for each possible transition from Baa. The first range is $\left(0, \hat{p}_{41}\right]$. Summing $\hat{p}_{41}$ and $\hat{p}_{42}$ gives the cumulative probability that the migration is either to Aaa or Aa and hence, the second range is $\left(\hat{p}_{41}, \hat{p}_{41}+\hat{p}_{42}\right]$. The third range is $\left(\hat{p}_{41}+\hat{p}_{42}, \hat{p}_{41}+\hat{p}_{42}+\hat{p}_{43}\right]$ and so forth. The daily rating $X_{1}$ is obtained by randomly drawing from a uniform distribution, $r \sim$ i.i.d.U[0,1], and matching this draw $\hat{r}_{\mathbf{1}}$ with one of the probability ranges. For instance, if $\hat{r}_{1} \in\left(\hat{p}_{41}+\hat{p}_{42}, \hat{p}_{41}+\hat{p}_{42}+\hat{p}_{43}\right]$ then $X_{1}=A$. Another random draw $\hat{r}_{2}$ gives $X_{2}$ and so forth over the sovereign's lifetime $h$ so that $\left\{X_{1}, \ldots, X_{h}\right\}$ is obtained. This simulation is conducted independently for the remaining $N-1$ sovereigns to construct the first bootstrap sample, $B_{1}$, that contains $N$ rating histories, each with length $h_{i}, i=1,2, \ldots, N$. This procedure is then iterated to obtain $R$ bootstrap data sets $\left\{B_{j}\right\}_{j=1}^{R}$. Finally, each $B_{j}$ is translated into $N$ rating transition histories and durations which, using equation (2), yield the DM one-year transition matrix estimate $\hat{P}_{j}-$ thus we obtain $R$ transition matrix estimates from the DM approach. Likewise, using equation (6) we obtain $R$ transition matrix estimates from the HHR approach. The empirical distribution of the DM and HHR mobility differential, $\left\{\widehat{\Delta m}_{j}\right\}_{j=1}^{R}$, is constructed in a similar manner. See Appendix A for details. ${ }^{4}$

The homogeneous (HHR) and non-homogeneous (NHHR) hazard rate estimators are compared through similar experiments. However, in order to introduce year-on-year heterogeneity in the DGP, we estimate annual intensity and probability matrices, $\hat{\Lambda}_{t}$ and $\hat{P}_{t}=\exp \left(\frac{1}{365} \hat{\Lambda}_{t}\right), t=1,2, \ldots, T$ on the basis of the observed

\footnotetext{
${ }^{3}$ There is no agreement in the credit risk industry on whether the ratings are cross-sectionally independent. S\&P (2001) argue that their rating transitions over the recent past are country specific. In this paper, we build on the evidence in Wilson (1997), Nickell et al. (2000), Christensen et al. (2004), and Stefanescu and Tunaru (2005) and conceptualize the rating histories of the various issuers as independent. While this assumption can be disputed, for the purpose of our analysis the key issue is to employ the same bootstrap design for all the estimators under study.

4 The paper appendices are downloadable from the author's website - web address to be inserted after the journal refereeing process is complete. The referees were sent copies of the Appendices.
} 
ratings. ${ }^{5}$ Then using $\hat{P}_{t}$, an artificial daily rating history is obtained (as detailed above) for each sovereign per year. In order for this heterogeneity to be reflected in the estimators' properties, a transition horizon $\Delta_{t}>1$ year is required. We choose $\Delta_{t}=2$ years as the horizon of interest and thus compute a sequence of HHR biannual transition matrices by deploying equation (6) over the 1982-1983, 1984-1985,..., 2002-2003 periods. Likewise, by means of equation (7), a sequence of NHHR matrices is obtained. Finally, the long run average formulae $(9)$ where $\left(t_{k}, t_{k+2}\right), k=0, \ldots, T-2$ are 2 -year periods is deployed with each sequence to construct a pair of 2-year transition matrices, the HHR and NHHR estimates, respectively.

The estimators' empirical distributions are used to construct confidence intervals and to test hypotheses. One can assess the estimators' finite-sample bias by comparing the mean of their distribution with the 'true' parameter value in the bootstrap DGP. Following Efron and Tibshirani (1993) we adopt $R=1000 .^{6}$

\section{The Data}

The data is from Moody's Default Risk Service. The credit ratings represent Moody's assessment on the risk that a borrower will fail to honour any debt payment — structural features of the debt issues such as maturity, coupon structure, collateralization and seniority are all taken into account. The sovereigns sampled are those with foreign-currency bonds rated by Moody's some time during March 5, 1981 through March 4, 2004. Thus the rating histories of the $N=72$ sovereigns in the sample have different lengths $h_{i}, i=1,2, \ldots, N$. The inputs for the discrete (DM) estimator are the credit ratings observed on the $5^{t h}$ March, taken as the start of each 12-month window, whereas the continuous (HHR, NHHR) estimators are based on the exact timing of each transition and the duration in each rating. Moody's occasionally assigns different ratings to bonds of the same issuer depending on characteristics. ${ }^{7}$ In order to convert the sovereign bond rating histories into a single sovereign rating history, we record for each issuer the lowest rating on senior unsecured bonds which have not yet matured or been repaid. The latter is the most meaningful indicator of a sovereign's likelihood of default on any of its bonds (Moody's, 2003).

According to Moody's, a sovereign default occurs when a country misses payments on any of its foreign-

\footnotetext{
${ }^{5}$ It seems sensible not to introduce heterogeneity across shorter periods than one year for two reasons. First, the generator $\hat{\Lambda}_{t}$ estimated over 6 or 4 month periods would be highly inaccurate because few sovereign transitions are generally observed in such short intervals. Second, the stylised stability of sovereign ratings suggests that within-year homogeneity is plausible.

${ }^{6}$ In order to assess whether 1000 replications guarantee convergence of the bootstrap, we performed two sets of 500 simulations. These gave empirical distributions that are virtually identical and comparable to that from the larger sample.

7For instance, Russia's 'MinFin' US dollar bonds have been generally rated lower than its Eurodollar bonds. During the 1999 Russian crisis, defaults occurred on the former but not on the latter.
} 
currency rated bonds. Moody's does not have a 'default' rating as such but instead records default dates the current rating (e.g. Caa) represents the severity of the default. For each country, we treat the date of the first default announcement as the default date — these are 17/08/98 (Russia), 30/11/98 (Pakistan), 25/08/99 (Ecuador) , 20/01/00 (Ukraine), 7/09/00 (Peru), 13/06/01 (Moldova), 30/11/01 (Argentina) and 15/05/03 (Uruguay). In order to identify the pre-default rating, we track the rating sequence up to the default date and throw out the transitions that occurred very close (within a month) to it. For instance, Ukraine was downgraded from Caa3 to Ca at day $t-15$ in default event time and so the pre-default rating is Caa3 as the downgrade clearly reflected a pending default. Appendix B reports the number of foreign-currency sovereign bond issuers rated by Moody's from 1981-2004 in Panel A and provides a breakdown according to issuer's characteristics (geographical location, state of the economy and credit quality) in Panel B. Unlike emerging economies, most industrialized countries have ratings from the start of the sample (March, 1981).

The initial date of the rating history or left censoring varies across sovereigns. The same applies to the termination date or right censoring. The latter can occur either at the end of the sample window (i.e. March 4, 2004) or at an earlier point due to withdrawn rating (WR) or default. A sovereign receives a WR when bonds have matured, been repaid or called. The latter often reflects the issuer's temporary exit from the public bond market rather than having negative credit implications (Carty, 1997). WRs are rather scarce because sovereigns, as opposed to corporates (due to merger or liquidation), rarely retire all their debt simultaneously - only 5 countries in the sample have experienced a WR. We follow the literature and exclude the WRs from the history of a sovereign (right censoring). When the rating is resumed, the latter will be treated as a new issuer. Likewise, any rating assigned between the default date and the end of the default episode is discarded. The sovereign is treated as a new issuer when the default episode ends, defined as the date when the sovereign exceeds the B3 rating. ${ }^{8}$ Half of the 8 defaulted countries (Pakistan, Peru, Russia and Ukraine) recover from default and reappear in our sample as new issuers whereas the rest (Argentina, Ecuador, Moldova and Uruguay) remain in default until the end of the sample window.

Finally, the analysis is focused on the eight ratings used by Moody's prior to 1982 (i.e. Aaa to C plus default and hence, a sub-rating such as Baal is labelled Baa) for two reasons. First, in the context of the sparsity of data for sovereigns this reduces the number of parameters to be estimated and increases the sample size of transitions per rating. Second, the reliability of finer rating transition estimates is questionable

\footnotetext{
${ }^{8}$ This procedure is equivalent to treating default as an absorbing state.
} 
in credit risk applications and so the coarse rating system has become the industry standard.

Thus the effective sample 1981-2004 for the analysis has 81 rating histories, 4 pertain to countries that re-emerged from default and 5 to countries with a WR. Figure 1 displays the distribution of aggregate ratings.

[Figure 1 around here]

Investment grade sovereigns represent $70 \%$ of the sample. Appendix C provides summary statistics on the distribution of credit ratings per year. Overall, the sample comprises a total of 759 country-year cases and 104 rating transitions. A discrete estimation approach (that ignores within-year migrations) exploits only 80 of these 104 cases which means discarding $23 \%$ of the observed migrations. ${ }^{9}$

\section{Properties of Transition Probability Estimators}

This section first analyzes the discrete and continuous estimators for a 1-year transition horizon. Second, the latter is increased to 2 and 3 years and the sample estimates are compared. Third, the added value of a time-heterogeneous approach to credit risk estimation is assessed. As explained in Section 2.5, for the latter purpose we introduce year-on-year heterogeneity in the bootstrap DGP and then consider 2-year transition matrices as the estimation object. The discussion focuses primarily on the probabilities of default (PD) for each rating and on the overall mobility or migration risk implied by the transition matrices. ${ }^{10}$

\subsection{Discrete versus Continuous Estimators}

\section{One-year Transition Horizon}

In the discrete (DM) approach, the entry $p_{i j}$ of the 1-year transition probability matrix $P$ is estimated through (2). In the continuous (HHR) approach, the entry $\lambda_{i j}$ of the intensity matrix $\Lambda$ is estimated through (5) and $\hat{P}$ is obtained as the matrix exponential of $\hat{\Lambda}$ (horizon $\Delta t=1$ year) for a $6^{t h}$ order Taylor expansion $(k=6)$. Table 1 reports the DM and HHR transition probabilities.

[Table 1 around here]

\footnotetext{
${ }^{9}$ The only empirical study available on sovereign transition matrix estimation, Hu et al. (2002), adopts a discrete-time framework and so it exploits only 26 of S\&P's rating transitions during 1981-1998.

${ }^{10}$ The main inputs for portfolio credit risk and capital allocation models are the default probability estimates.
} 
The DM estimates are very similar to those reported by Moody's (2003). Both the DM and HHR matrices are diagonally dominant (rating stability) implying that the migrations are typically towards neighbouring ratings. Larger volatility (transition between distant ratings) is associated with lower credit ratings such as $\mathrm{Ba}, \mathrm{B}$ and $\mathrm{C}$. Another common aspect is that for any given rating $i$ (or $i$ th row) the transition probabilities decrease as one moves farther from the diagonal - this is known as row monotonicity and is a stylized feature of credit rating migration matrices (JP Morgan, 1997; p.73). A violation of monotonicity occurs for the $\mathrm{B}$ rating: there is a higher probability of migrating to default than to $\mathrm{C}$. This perverse effect, which is more prominent in the DM matrix, may stem from the noisy nature of the data for the low $\mathrm{B}$ rating.

The HHR estimator yields non-zero probabilities for transitions that are absent in-sample whereas for such cases, the coarser DM estimator yields zero by construction. In the HHR matrix the off-diagonal probability mass is spread over nearly all ratings whereas in the DM matrix it is concentrated around the diagonal. In particular, it is noticeable that for low ratings the HHR transition matrix reflects greater migration volatility than the DM counterpart. These differences suggest that the continuous estimator better captures the rating dynamics. Moreover, the default probability for rating $\mathrm{C}$ in the DM matrix $\left(\hat{p}_{C, D}=16.7 \%\right)$ is relatively low as compared to that in the HHR matrix (28.9\%). This difference can be explained by the fact that the coarse DM estimator does not use information on rating duration. For instance, if a sovereign is rated B at year beginning then is downgraded to $\mathrm{C}$ and eventually ends the year in default, the discrete estimator will only account for the overall migration from $\mathrm{B}$ to $\mathrm{D}$. In contrast, the full transition history $\mathrm{B} \rightarrow \mathrm{C} \rightarrow \mathrm{D}$ together with the duration in $\mathrm{C}$ will be exploited by the continuous estimator. The duration in $\mathrm{C}$ is short (in most sample cases below 1 year as this is just a transitional status toward D) which implies a large default intensity $\hat{\lambda}_{C, D}$ and hence, this explains the relatively large HHR default probability for rating C.

Next we compare the small-sample properties of the DM and HHR estimators through the bootstrap framework presented in Section 2.5. The bootstrap DGP is a time-homogeneous, continuous Markov chain with 'true' parameter values the historical (HHR) transition probabilities. Since the main focus is on default risk, the last column of the transition matrix (Table 1) is highlighted in bold to signify the true probabilities of default (PD). Figure 2 plots the Gaussian kernel density estimate of the simulated PDs for investment grade ratings. The bold vertical line signifies the true PD and the two dashed lines indicate the $95 \%$ confidence interval. For the three top ratings (Aaa, Aa, A), the estimates from the coarse DM approach are zero for all bootstrap rating histories and so are not plotted. Table 2 reports the true PDs in the bootstrap Markov 
chain alongside summary statistics for the empirical distribution of the DM and HHR estimators.

[Figure 2 and Table 2 around here]

For the three top ratings, the distribution of the HHR estimator is roughly exponential with 97.5 th percentiles of $1.23 \times 10^{-5} \mathrm{bp}, 0.0009 \mathrm{bp}$ and $0.238 \mathrm{bp}$, respectively, and means quite close to the true PDs (small bias). Although direct transitions to D from top ratings are unobserved in the current (and bootstrap) histories, the HHR estimator is able to provide a plausibly small but non-zero probability for such rare events. Regarding rating Baa, the DM estimator produces a zero PD for most histories and a PD in the range [60, 125]bp for a few histories (Figure 2) which leads to a rather high 97.5 th percentile of $75.2 \mathrm{bp}$. In sharp contrast, the 97.5th percentile for the HHR estimator is $9.72 \mathrm{bp}$, suggesting a marked efficiency gain as compared to the coarser DM estimator. ${ }^{11}$ Our analysis serves to assess the adequacy of the minimum probability at $3 \mathrm{bp}$ that has been established by the Basel Committee for unobserved events. This probability clearly falls in the 95\% confidence interval of the HHR estimator for rating Baa while it is well beyond the 97.5th percentile for Aaa, Aa and A. Hence, the Basel II threshold appears too conservative for these higher ratings.

The empirical distribution of the $\mathrm{PD}$ for the lower ratings $\mathrm{Ba}, \mathrm{B}$ and $\mathrm{C}$ are shown in Figure 3 .

[Figure 3 around here]

Direct defaults from B are the most likely and relatedly, the DM estimator has a smooth distribution only for rating B. For rating B, the DM and HHR estimators have a $95 \%$ confidence interval of similar width but the latter is more efficient (tighter confidence interval) for ratings Ba and C. The trimodal distribution of the HHR estimator for Ba may be because there are three main transitions from Ba in the sample. One is the $\mathrm{Ba} \rightarrow$ Baa upgrade with $6.76 \%$ probability, another is the $\mathrm{Ba} \rightarrow \mathrm{B}$ downgrade with $8.55 \%$ probability and third, a direct default with $0.08 \%$ probability. Since these probabilities are the true parameter values in the bootstrap DGP, simulated histories following the upgrade (downgrade) contribute to the left (right) peak of the PD distribution and those with direct default transition contribute to the middle peak. Regarding small-sample bias in the PD parameters (difference between the distribution's median and the bootstrap DGP parameter), the HHR estimator shows less bias than the DM estimator for high credit-quality ratings and rating $\mathrm{C}$ whereas the opposite holds for ratings $\mathrm{Ba}$ and $\mathrm{B}$.

\footnotetext{
${ }^{11}$ The higher DM percentile is because about $2.5 \%$ of the simulated paths contain at least one default from Baa. If one default occurs, then the DM probability in simulation $j$ is expected to be $1 / N_{B a a}^{j}$ where $E\left(N_{B a a}^{j}\right)=112$ and $1 / 112$ is roughly 75.2 bp.
} 
We now assess the statistical significance of differences in the DM and HHR default probabilities. For this purpose, we conduct two-sided bootstrap tests for $H_{0}: P D_{H H R}-P D_{D M}=0$. Table 3 (Panel A) summarises the empirical distribution of the differential statistic $\left(\widehat{P D}_{H H R}-\widehat{P D}_{D M}\right)$.

[Table 3 around here]

The 95\% confidence interval contains zero thus suggesting insignificant differences. The exception is rating A for which the DM estimator gives always a zero default probability because no direct transitions $\mathrm{A} \rightarrow \mathrm{D}$ are observed in the sample. However, the more efficient HHR estimator gives a non-zero probability because it exploits the full information that there are transitions $\mathrm{A} \rightarrow \mathrm{Ba}$ and $\mathrm{A} \rightarrow \mathrm{Baa}$ together with $\mathrm{Ba} \rightarrow \mathrm{D}$ and $\mathrm{Baa} \rightarrow \mathrm{D}$. These findings suggest that the DM estimator may underperform the HHR estimator for some mid ratings.

Finally, we test for significant differences in the overall ratings mobility (or migration risk) implied by the DM and HHR estimation methods. Table 3 (Panel B) provides the historical mobility differential, $\widehat{\Delta m}$, as well as summary statistics for the estimator's bootstrap distribution. The migration risk implied by the DM transition matrix is higher than that from the HHR matrix and the difference is statistically significant as suggested by the $95 \%$ confidence interval not containing zero. This suggests that the concentration of probability mass at off-diagonal positions in the DM transition matrix is higher than that in the HHR counterpart. Upon closer inspection of Table 1, it is apparent that the DM matrix is relatively more sparse (large number of zero entries) and, in particular, the mass is largely concentrated in those few ratings for which transitions have been observed in the sample (i.e. around the diagonal). These results are consistent with the discussion in Section 2.4, namely, that a few large off-diagonal terms can inflate the $m(\tilde{P})$ metric considerably. The HHR matrix has not only higher default probability mass (sum of last column entries) than the DM matrix but this mass is also spread over a larger number of ratings.

\section{Two-year and Three-year Transition Horizon}

One would expect a larger contrast between the DM and HHR estimators as the transition horizon increases because the latter captures better the more marked rating activity. Table 4 presents the probability estimates.

[Table 4 around here]

The differences are striking for the lower ratings — the DM estimate suggests zero default risk from rating 
$\mathrm{C}$ whereas the HHR probabilities are $38.18 \mathrm{bp}$ and $35.49 \mathrm{bp}$ for the 2 - and 3 -year horizons, respectively. ${ }^{12} \mathrm{~A}$ non-zero probability of default from $\mathrm{C}$ over a relatively long horizon of 2 or 3 years is rather plausible. The upshot is that the DM approach underestimates the default probability for the highly risky sovereigns. On the other hand, the DM estimate of the default probability from Baa is about twenty-fold the HHR estimate — the 2-year default probabilities from the DM and HHR estimator are 192bp and 9bp and the 3-year ones are $333 \mathrm{bp}, 22 \mathrm{bp}$, respectively. The smaller default risk for Baa suggested by the HHR estimator is more plausible because sovereigns spend relatively long times in the mid Baa rating (large duration as compared to other ratings) on their way up and down the rating scale - the latter is captured in the denominator of equation (5) which pulls down the default probability for Baa. Regarding overall mobility, it turns out that the DM estimator yields relatively high measures, $m(\tilde{P})$, as compared with the HHR estimator for all horizons ranging from 1 to 3 years. Again this stems from the large concentration of probability mass in a few off-diagonal positions. Unsurprisingly, the differential $\widehat{\Delta m}\left(\tilde{P}_{D M}, \tilde{P}_{H H R}\right)$ increases with the time horizon.

These differences are just a reflection of pitfalls in discrete versus continuous estimation of rating migration risk, namely, larger small-sample biases and inefficiency of the former because of neglecting information on finer migration activity (within the horizon of interest) and rating durations. In sovereign credit risk analysis, where ratings data are very sparse, it is crucial to account for as much information as possible.

\subsection{Homogeneous versus Heterogeneous Estimators}

The importance of allowing for time heterogeneity in the estimation of sovereign rating-transition probabilities has not been investigated as yet. To fill this gap, we compare the homogeneous (HHR) and nonhomogeneous (NHHR) hazard rate estimators. In order to approximate the estimators' sampling distribution, bootstrap rating histories are obtained from a DGP with year-on-year heterogeneity as described in Section 2.5 - the longer the transition horizon $\Delta_{t}$, the more prominent the time heterogeneity will be and so the estimation focuses on 2-year and 3-year transition matrices. Accordingly, a sequence of biannual transition matrices is obtained by deploying equation (6) over non-overlapping 2-year periods and the HHR transition matrix is constructed via the issuer-weighted long run average $(9)$. We proceed similarly over 3 -year periods to construct the HHR estimate of the 3-year transition matrix and so forth. Likewise, we obtain the NHHR

\footnotetext{
${ }^{12}$ The unit probability estimates from the DM approach, $\hat{p}_{C, B}=1$ and $\hat{p}_{C, B a}=1$ (for the 2- and 3-year horizons, respectively), stem from one transition - Romania migrates from $\mathrm{C}$ to $\mathrm{B}$ after 2 years and to Ba after 3 years. All other C-rated issuers have durations shorter than 2 years and so their rating dynamics cannot be captured by the coarse discrete estimator.
} 
estimate of the 2-year and 3-year transition probability matrices by first deploying equation (7) sequentially and then by averaging the matrix sequences through (9). As explained in Section 2, the NHHR estimator captures heterogeneities within the estimation window $\left(\Delta_{t}\right)$ whereas the HHR estimator neglects them.

Table 5 reports the HHR and NHHR transition probability estimates and overall mobility measures.

[Table 5 around here]

All credit ratings are subject to greater migration according to the NHHR matrix - larger off-diagonal probability mass in each row or equivalently, per rating. In particular, the NHHR estimator generally implies greater default risk. As one would expect, increasing the transition horizon exacerbates the differences between the HHR and NHHR default probabilities. The $\widehat{\Delta m}$ statistic increases with the transition horizon suggesting also that the contrast in the implied migration risk widens with the estimation window.

We now compare the bootstrap distribution of the two estimators. To preserve space, the discussion focuses on the 2-year probabilities of default (PD). Figure 4 reports the kernel density of the estimated probabilities (over replications) for investment-grade issuers. The summary statistics are in Table 6.

[Figure 4 and Table 6 around here]

For ratings Aaa and Aa, the estimated PDs are zero for all bootstrap samples and so the kernel density is not plotted - the true PDs are zero because neither direct nor indirect transitions to default are observed over 2-year periods in the actual (Moody's) sample. ${ }^{13}$ For ratings A and Baa, the true PDs are non-zero and both estimators produce smooth distributions. The NHHR estimator yields zero default probabilities for rating A with most simulated samples and just a few probabilities in the range $(0,2] \mathrm{bp}$, giving a $97.5 \%$ confidence band of $0.45 \mathrm{bp}$. Similarly, the corresponding percentile for the HHR estimator is $0.43 \mathrm{bp}$, rendering the two comparable in terms of efficiency. For the Baa category, the $95 \%$ confidence interval is again virtually identical for both estimators. For these two ratings, the comparison in terms of bias gives mixed results depending on which measure (mean or median) is used.

Figure 5 plots the kernel density of the simulated PDs for non-investment grade issuers.

[Figure 5 around here]

\footnotetext{
${ }^{13}$ The true PDs are obtained from Moody's sample by first estimating annual transition matrices using (5) and (6). Second, a sequence of 2-year transition matrices is obtained by multiplying each consecutive pair of annual matrices. The long run average matrix obtained from this sequence through (9) provides the parameter values, or true PDs, for the bootstrap DGP.
} 
For the NHHR estimates, the densities are smooth and centered at the true value (c.f. true PD and mean PD; Table 6) with the exception of rating C. In contrast, the density of the HHR estimates suggests relatively large bias - the true PD is on the right tail. This vindicates that neglecting heterogeneity may lead to downward biases in default risk measures for speculative ratings. However, the HHR estimator produces tighter confidence intervals than the NHHR estimator. For rating $\mathrm{C}$, the sparse transition data within the 2-year windows leads to uncentered distributions with wide confidence intervals for both estimators.

Table 7 reports summary statistics for the bootstrap distribution of the differential $\left(\widehat{P D}_{H H R}-\widehat{P D}_{N H H R}\right)$.

[Table 7 around here]

For rating $\mathrm{B}$, the $95 \%$ confidence set does not contain zero thereby suggesting that the HHR and NHHR estimates imply significantly different default risk - the HHR estimator underestimates the PD for rating B. The mean/median estimates are similar for all other ratings. In terms of overall mobility or migration risk, the two estimates are also significantly different. In particular, the mobility implied by the NHHR matrix is significantly larger or equivalently, it contains more off-diagonal probability mass than the HHR matrix and so the inferred migration risk from the latter is smaller. The upshot is that, if there is time heterogeneity in the ratings DGP, disregarding it may produce misleading inferences on migration risk.

\section{Properties of the Sovereign Rating Process}

Transition matrix estimators typically assume that the credit ratings are driven by a Markov process. To the best of our knowledge, the plausibility of the latter has not been assessed for sovereigns. In this section, we attempt to fill this gap in two ways. First, we test whether the transition matrix estimate conforms to the homogeneous Markov structure. Second, we investigate the presence of momentum and duration effects both of which are at odds with the Markovian assumption and induce time heterogeneity in the rating process.

\subsection{Does the Transition Matrix Conform to the Markov Structure?}

Each row of the transition matrix $P$ sums to one and so it follows that there exists a trivial unity eigenvalue $\left(q_{1}=1\right)$ which is the largest in magnitude - the rating process reaches a steady state as time goes to infinity. The decay rate towards the steady state is dictated by the second largest eigenvalue $\left(q_{2}\right)$. It can be shown that the eigenvector associated with $q_{2}$ characterizes the asymptotic distribution of survivors (sovereigns not 
ending in default) and thus provides insights on their limit rating. For a process to be homogeneous Markov, the eigenvalues of $P$ associated with increasing horizons should decay exponentially (except for $q_{1}$ which is unity for all horizons) and the eigenvectors should remain constant. In other words, if $q_{2}$ is the 2nd largest eigenvalue of $P$ for transition horizon $\Delta t$, then $\ln \left(q_{2}\right)=-C \Delta t$ for $C>0$. This $\log$-linear relation has to hold for all subsequent eigenvalues if the rating process driving $P$ is Markov and time-homogeneous.

The second to fifth largest eigenvalues of $\hat{P}$ for various horizons are plotted in Figure 6 (Panel A). The transition matrices are estimated using equation (6). The graph strongly supports the log-linear relation.

[Figure 6 around here]

Figure 6 (Panel $\mathrm{B}$ ) shows that the 2nd largest eigenvector of $\hat{P}$ is very similar across horizons. Moreover, the distribution peaks at Aaa which suggests that the survivors tend to settle (long term) at the highest rating. On the basis of this analysis, there is no evidence against the Markovian assumption.

\subsection{Do Ratings Display Duration and Momentum Effects?}

The duration dependence hypothesis says that the time a sovereign stays in a given rating impacts the future probability of transition to another rating. To test this hypothesis, panel logit models are estimated using monthly sovereign credit ratings and durations over 23 years (March, 1981 to March, 2004). The duration $d_{i t}$, recorded for each sovereign $i$ at the end of every month $t$, is the time elapsed since the last transition to the current rating. To illustrate, consider a sovereign that experienced a rating transition to Ba in June 2002, then to B in September 2003 and has not moved since then. The duration in, say, June 2003 is 12 months and in March 2004 it is 6 months. The rating process is assumed to start in March 1981 (left-censored durations) which is not too restrictive because very few issuers had been rated before 1981 . The duration hypothesis is assessed separately for upgrades (UP) and downgrades (DW) by defining the dummy variable

$$
U P_{i t}= \begin{cases}1 & \text { if sovereign } i \text { was upgraded in month } t \\ 0 & \text { otherwise }\end{cases}
$$

and likewise for $D W_{i t}$. The following logit regression is estimated for upgrades

$$
y_{i t}^{*}=\alpha+\beta x_{i t}+\gamma^{\prime} \mathbf{z}_{t}+\varepsilon_{i t}, \varepsilon_{i t} \sim i i d\left(0, \sigma_{i}^{2}\right), \quad i=1, \ldots, N, \quad t=1, \ldots, T
$$

where $y_{i t}^{*}$ is a continuous latent variable such that $U P_{i t}=1$ for $y_{i t}^{*} \geq 0$ and $U P_{i t}=0$ otherwise (likewise for $\left.D W_{i t}\right), x_{i t} \equiv d_{i t}$ and $\mathbf{z}_{t}$ is $7 \times 1$ vector of yearly dummies 1998-2004 to signify that many emerging 
economies entered the sample after 1997. This model allows for heteroskedasticity because the error variance will be relatively smaller for industrialized countries if their durations are more stable — the error variance is permitted to differ between industrialized and non-industrialized countries according to $\sigma_{i}=\left[\exp \left(\psi+\xi r_{i t}\right)\right]$ where $\xi$ is a constant parameter and $\exp (\psi)=\frac{\pi}{\sqrt{3}}$ with $r_{i t}=1$ if $i$ is industrial and $r_{i t}=0$ otherwise. The observed durations reveal that: a) the mean of $d_{i t}$ increases with rating quality, the only exception being the absorbing default rating, b) the variance of $d_{i t}$ for top credit-quality ratings is relatively large but this is a scale effect (long durations) as the standard deviation/mean ratio bears out. For details see Appendix D.

Table 8 (Panel A) reports the maximum likelihood estimation results for the above logit model

[Table 8 around here]

The duration coefficient is negative for both upgrades $(\hat{\beta}=-1.88)$ and downgrades $(\hat{\beta}=-1.66)$ which suggests that migration probabilities are negatively influenced by duration — the longer a sovereign stays in a given rating the less likely a migration is. These findings are consistent with those in Lando and Skodeberg (2002) for corporates and can be attributed to the rating agencies' reluctancy to take drastic actions in favour of gradual (notch-by-notch) upgrades/downgrades. The latter results in short durations and high migration risk for the low end of the rating scale and vice versa. Finally, note that although the estimated error variance is higher for industrial countries this finding is insignificant — the positive sign of $\hat{\xi}$ may relate to the high durations typical of top-quality ratings but, in standardized form, the durations are comparable across ratings which explains the insignificance of $\hat{\xi}$ (for details see Appendix D).

The rating momentum hypothesis states that a sovereign being upgraded (downgraded) is more susceptible to subsequently being upgraded (downgraded). To test this hypothesis for upgrades, we define the upward momentum indicator over the last 24 months

$$
U M_{i t}= \begin{cases}1 & \text { if sovereign } i \text { was upgraded to the current rating over }[t-1, t-23] \\ 0 & \text { otherwise }\end{cases}
$$

and (11) is estimated using $x_{i t} \equiv U M_{i t}$. Likewise for downgrades.

The results are presented in Table 8 (Panel B). The downgrade logit model strongly supports the momentum hypothesis with a highly significant $\hat{\beta}$ at 1.20 . This suggests that a downgrade in the previous two years significantly increases the current downgrade probability. These findings are in line with the extant evidence for corporate debt (Lando and Skodeberg, 2002; Nickell et al., 2000). Moreover, the residual variance is 
significantly lower for industrialized countries $(\hat{\xi}=-0.17)$ which is in line with their more stable rating history. The upgrade logit provides, however, no evidence of momentum.

What do we learn from these findings? On the one hand, the spectral analysis of the transition matrix $\hat{P}$ provided no evidence against the assumption of Markovian ratings. On the other, the panel logit models uncovered significant duration and momentum effects in sovereign ratings both of which imply non-Markovian behaviour and non-time homogeneity as the current rating does not fully determine the transition probability. Recent contributions in the corporate credit risk literature have introduced refinements into hazard rate estimators in order to accommodate momentum and duration effects (Kavvathas, 2001; Christensen et al., 2004; Koopman et al., 2005). However, these sophisticated estimators have not been applied in the context of sovereigns as yet and this may be because of data limitations.

\section{Conclusions}

Sovereign credit ratings play a major role in modern credit risk management, valuation and capital allocation. All these applications require transition matrices, namely, the default probabilities from each rating category and the probabilities of transition between them. Different estimators of transition matrices have been proposed, however, their behaviour has been studied mainly in the context of corporate ratings. Very little is known on the finite-sample properties of sovereign rating transition estimators. This paper contributes to the literature in three directions. First, it compares three alternative estimators that assume a Markov process for ratings - the discrete multinomial approach (industry standard) and two continuous hazard rate approaches that differ in how they deal with time heterogeneity. The discrete multinomial approach is attractive because of its computational simplicity while the time-heterogeneous hazard rate approach lies at the other extreme in terms of computational cost. The comparison is conducted through a parametric bootstrap technique that provides the estimators' empirical distribution. Second, we assess the validity of the assumption that the rating evolutions are Markov and time homogeneous. For this purpose, we rely on the spectral decomposition of the transition matrix estimates on the one hand, and on panel logit models to specifically test for rating momentum and duration effects on the other.

The analysis is based on a sample of Moody's credit ratings 1981-2004 for 72 industrialized and emerging economies. The default probabilities from continuous-time estimators are more reliable (smaller sampling variability) and generally less biased that those from the discrete estimator. Significant differences are 
also found in the overall migration risk implied by the estimators. The discrete transition matrices have a larger concentration of probability mass around the main diagonal which may misleadingly suggest a high degree of rating stability or low migration risk. This can be attributed to their efficiency loss in neglecting the full information of exact transition dates and rating durations. There are also differences between the homogeneous and heterogeneous hazard rate estimators and more so the wider the estimation window the longer the transition horizon the more noticeable the time heterogeneities become. The heterogenous estimator appears less biased than the homogeneous counterpart for the default risk of non-investment grade ratings. The default probabilities from the homogeneous estimator are downward biased. Interestingly, in terms of sampling variability there are no gains in using the time-heterogeneous estimator.

The improved understanding of the finite-sample behaviour of transition probability estimators has consequences for a number of issues in credit risk management and capital allocation. For instance, the Basel Committee recently established a lower bound of $3 \mathrm{bp}$ as the minimum transition probability for rare events. Our findings suggest that continuous estimators provide a more robust indication of the appropriateness of such a limit, in particular, suggesting that it is rather conservative (too high) for the top credit-quality sovereigns. Another relevant finding for financial institutions and regulators, in the light of the New Basel Accord, is that the choice between alternative estimators of sovereign default probabilities may be far from innocuous in terms of the implied capital requirements. However, further research is warranted to establish that the statistical differences here uncovered have economic significance.

There is evidence of non-Markov effects in the sovereign rating evolution. Panel logit models suggest negative duration dependence for both downgrades and upgrades. Rating momentum is significant for downgrades but not for upgrades in line with the rating agencies' practice of gradually reducing the credit quality status. These findings have important implications for risk management. For instance, in terms of pricing credit sensitive instruments, the rating duration and momentum of a sovereign may help to predict the value of its debt obligations. Hence, an issue that warrants investigation is whether duration and momentum are priced in sovereign debt or alternatively, whether there is scope for arbitrage. Finally, it may be fruitful to assess the incremental value of more refined estimators that account for momentum and duration effects in sovereign ratings. In particular, there is the issue of whether such estimators are feasible, given the sparse rating transition data for sovereigns, and if so what are the efficiency gains. 


\section{References}

[1] Aalen, O.O. and S. Johansen (1978) An empirical transition matrix for nonhomogeneous Markov chains based on censored observations. Scandinavian Journal of Statistics, 5, 141-150.

[2] Altman, E.I. (1998) The importance and subtlety of credit rating migration. Journal of Banking and Finance, 22, 1231-1247.

[3] Altman, E.I. and D.L. Kao (1992) The implications of corporate bond rating drift. Financial Analysts Journal, May/June, 64-75.

[4] Bangia, A., F.X. Diebold, A. Kronimus, C. Schagen and T. Schuermann (2002) Ratings migration and the business cycle, with applications to portfolio stress testing. Journal of Banking and Finance, 26 , $445-474$.

[5] Cantor, R., and F. Packer (1996) Determinants and Impact of Sovereign Credit Ratings, FRBNY Economic Policy Review, October, 37-52.

[6] Carty, L.V. and J.S. Fons (1993) Measuring changes in corporate credit quality. Moody's Special Report, Moody's Investors' Service, New York.

[7] Christensen, J., E. Hansen and D. Lando (2004) Confidence sets for continuous-time rating transition probabilities. Working Paper, University of Copenhagen.

[8] Geweke, J., R. C. Marshall and G. A. Zarkin (1986) Mobility indices in continuous-time Markov chains. Econometrica, 54, 1407-1423.

[9] Hu, Y-T., R. Kiesel and W. Perraudin (2002) The estimation of transition matrices for sovereign credit ratings. Journal of Banking and Finance, 26, 1383-1406.

[10] Israel, R. B., S. Rosenthal and J. Z. Wei (2001) Finding generators of Markov chains via empirical transition matrices, with applications to credit ratings. Mathematical Finance, 11, 245-265.

[11] Jackson, P. and W. Perraudin (2000) Regulatory implications of credit risk modelling. Journal of Banking and Finance, 24, 1-14.

[12] Jafry, Y. and T. Schuermann (2004) Measurement, estimation and comparison of credit migration matrices. Wharton Financial Institutions Center Working Paper. 
[13] Jarrow, R. A., D. Lando and S. M. Turnbull (1997) A Markov model for the term structure of credit risk spreads. The Review of Financial Studies, 10, 481-523.

[14] JP Morgan (1997) Creditmetrics - Technical Document. JP Morgan Discussion Paper, New York.

[15] Kalbfleisch, J. D. and R. L. Prentice (2002) The Statistical Analysis of Faihure Time Data, John Wiley \& Sons, New York.

[16] Kavvathas, D. (2001) Estimating credit transition probabilities for corporate bonds. Working Paper, University of Chicago .

[17] Kijima, M. and K. Komoribayashi (1998) A Markov chain model for valuing credit risk derivatives. The Journal of Derivatives, Fall, 97-108.

[18] Koopman, S. J., A. Lucas and A. Monteiro (2005) The multi-state latent factor intensity model for credit rating transitions. Working Paper, Vrije Universiteit Amsterdam.

[19] Lando, D. and T. Skodeberg (2002) Analyzing ratings transitions and rating drift with continuous observations. Journal of Banking and Finance, 26, 423-444.

[20] Moody's Investors Service (2003) Sovereign bond defaults, rating transitions and recoveries (1985-2002). Special Comment February.

[21] Nickell, P., W. Perraudin and S. Varotto (2000) Stability of Rating Transitions, Journal of Banking and Finance, 24, 203-227.

[22] Norris, J. R. (1997) Markov Chains, Cambridge University Press.

[23] Shorrocks, A. F (1978) The measurement of mobility. Econometrica, 46, 1013-1024.

[24] Stefanescu, C. and R. Tunaru (2005) Calibration of Default Probabilities to External Ratings. London Business School, Mimeo.

[25] Wei, J. Z. (2003) A Multi-factor, Credit Migration Model for Sovereign and Corporate Debts. Journal of International Money and Finance, 22, 709-735.

[26] Wilson, T. (1997) Credit Risk Modelling: A New Approach. Technical report, McKinsey Inc., New York. Mimeo. 


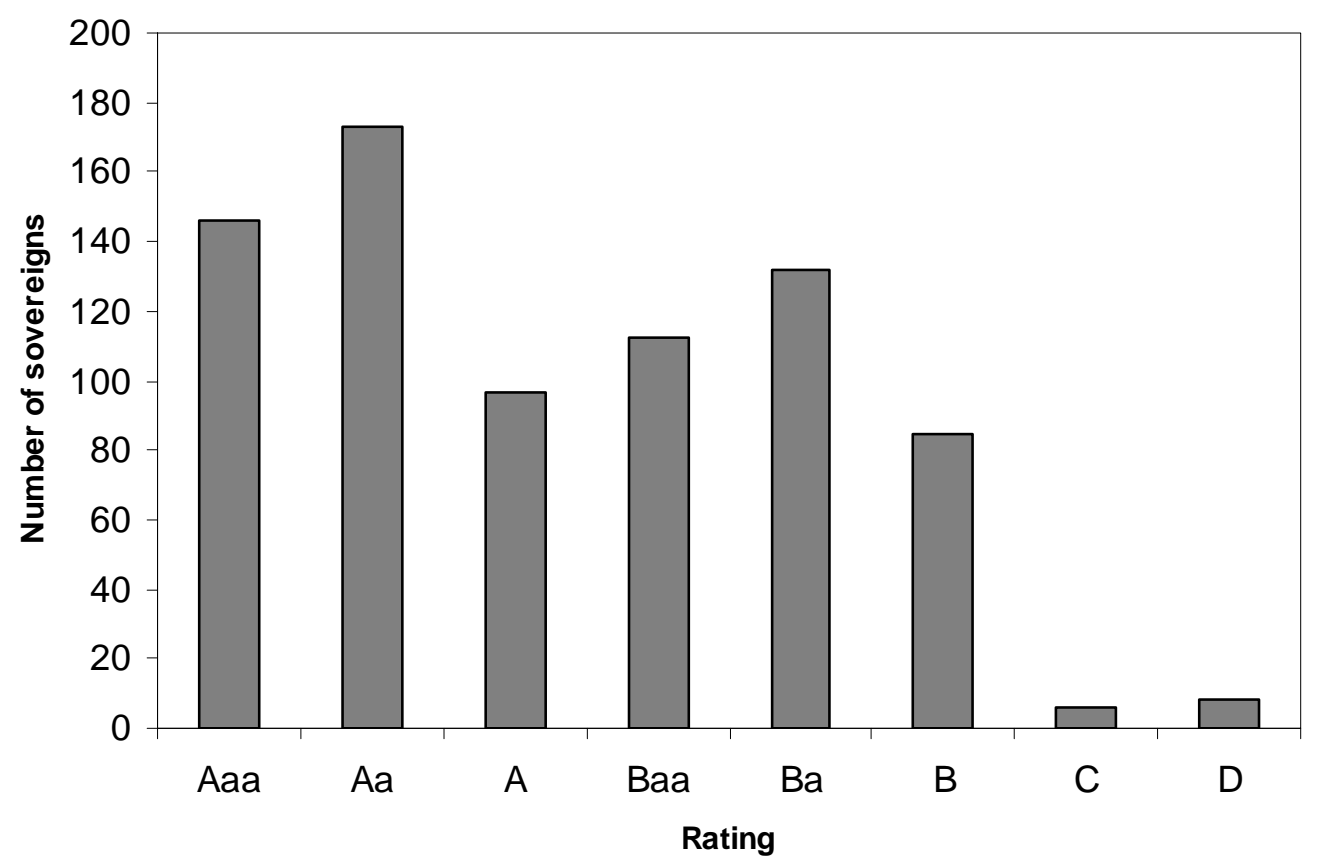

Figure 1. Distribution of Observed Sovereign Ratings

The figure shows the distribution of the sample (Moody's) ratings aggregated over all issuers $(N=81)$ and years $(1981-2004)$. 
HHR estimator

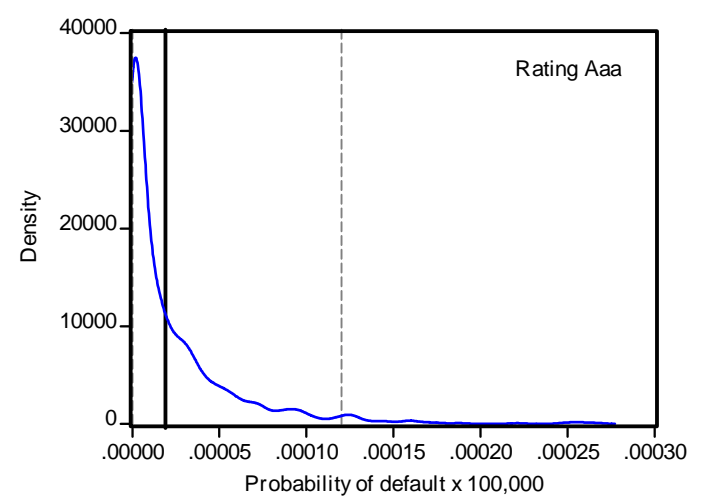

DM estimator

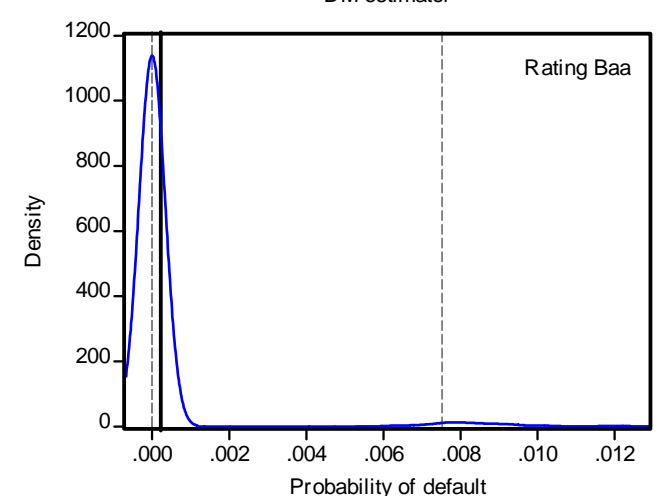

HHR estimator

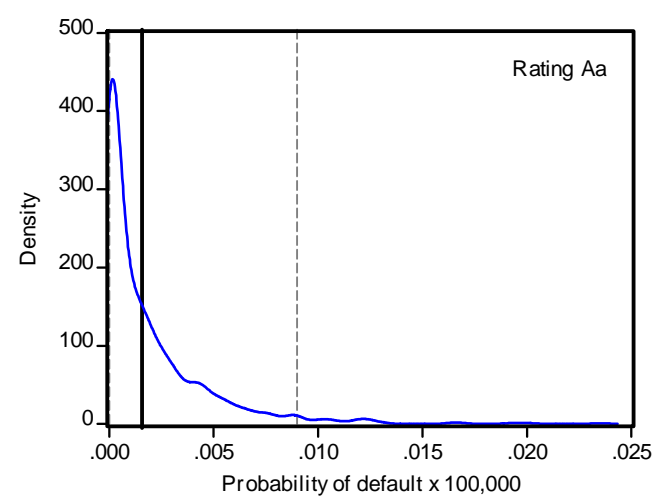

HHR estimator

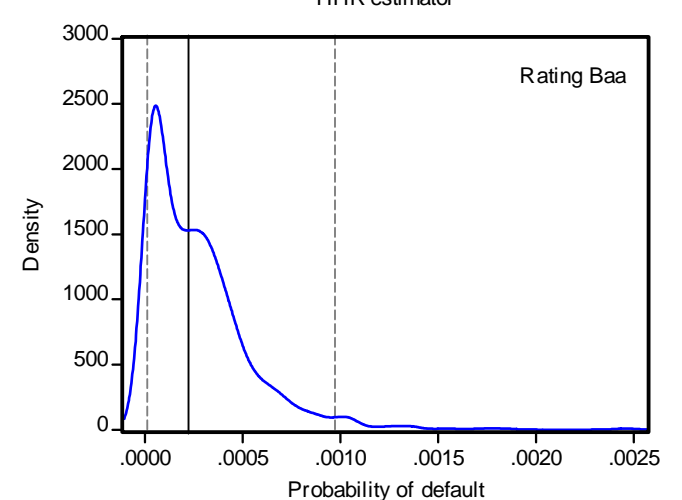

HHR estimator

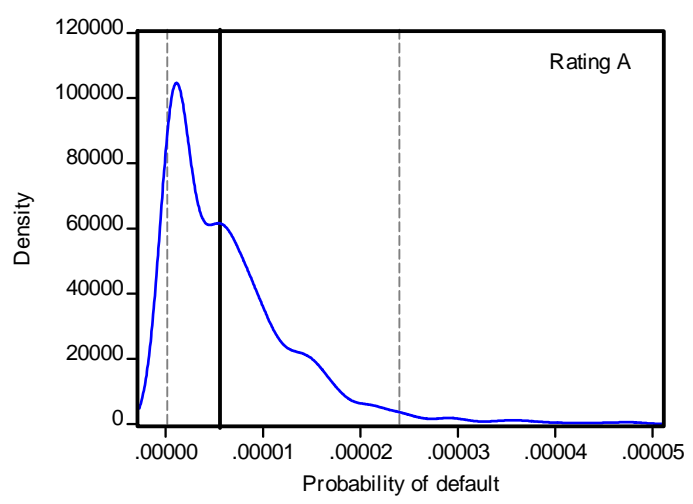

Figure 2. Empirical Distribution of Continuousand Discrete Default Probability Estimators(Investment Grade Ratings) The figure reports the Gaussian kernel density estimate of the simulated def ault probability for the continuous homogeneous hazard rate (HHR) estimator and the discrete multinomial (DM) estimator. 

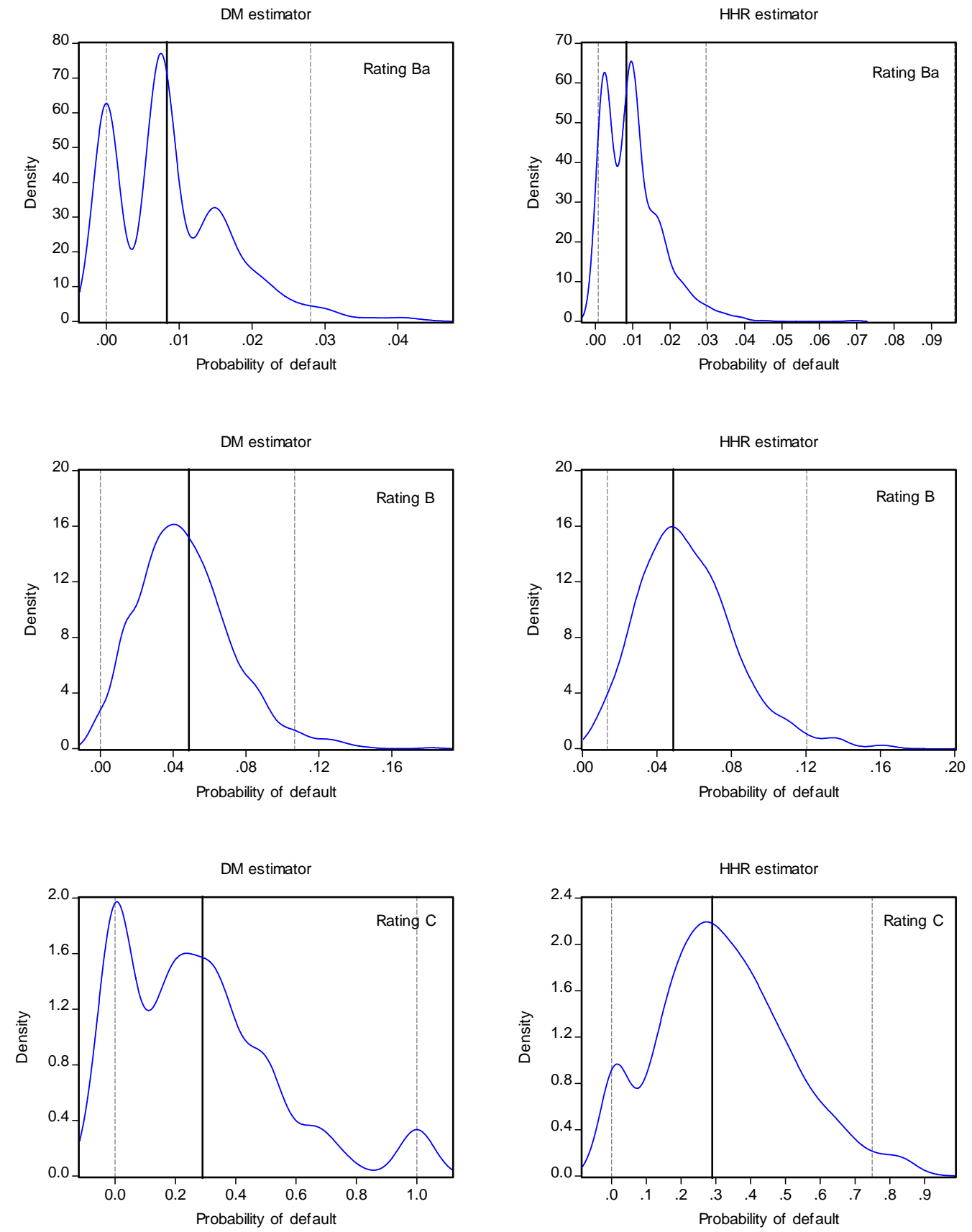

Figure 3. Empirical Distribution of Continuous/Discrete Default Probability Estimators (Non-investment Grade) The figure reports the Gaussian kernel density estimate of the simulated default probability for the continuous homogeneous hazard rate (HHR) estimator and the discrete multinomial (DM) estimator. 

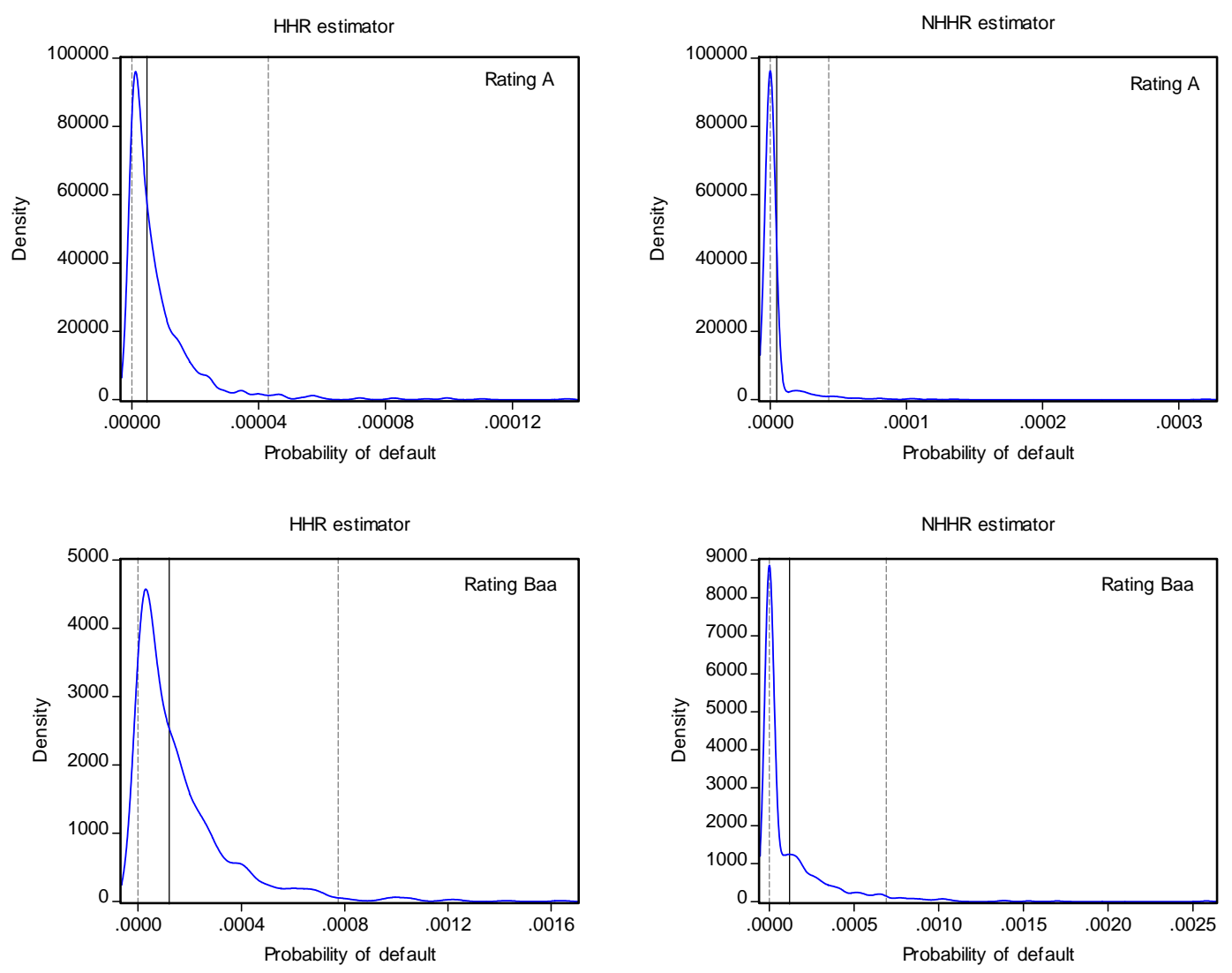

Figure 4. Empirical Distribution of Continuous Default Probability Estimators (Investment Grade Ratings)

The figure reports the Gaussian kernel density estimate of the simulated default probability for the continuous homogeneous hazard rate (HHR) estimator and the non-homogeneous hazard rate (NHHR) estimator. 

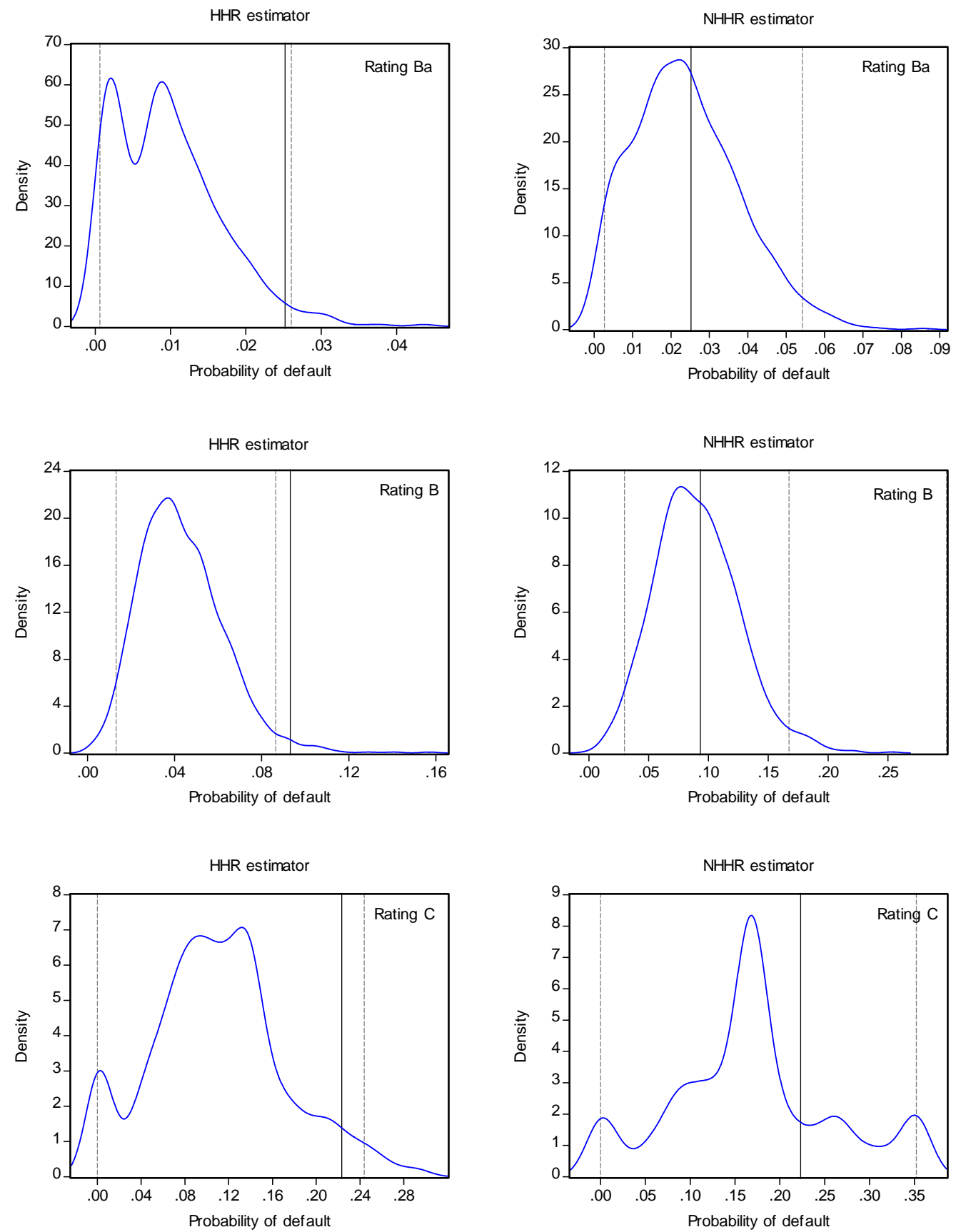

Figure 5. Empirical Distribution of Continuous Default Probability Estimators (Non-Investment Grade) The figure reports the Gaussian kernel density estimate of the simulated default probability for the continuous homogeneous hazard rate (HHR) estimator and the non-homogeneous hazard rate (NHHR) estimator. 
Panel A: Eigenvalues versus Transition Horizon

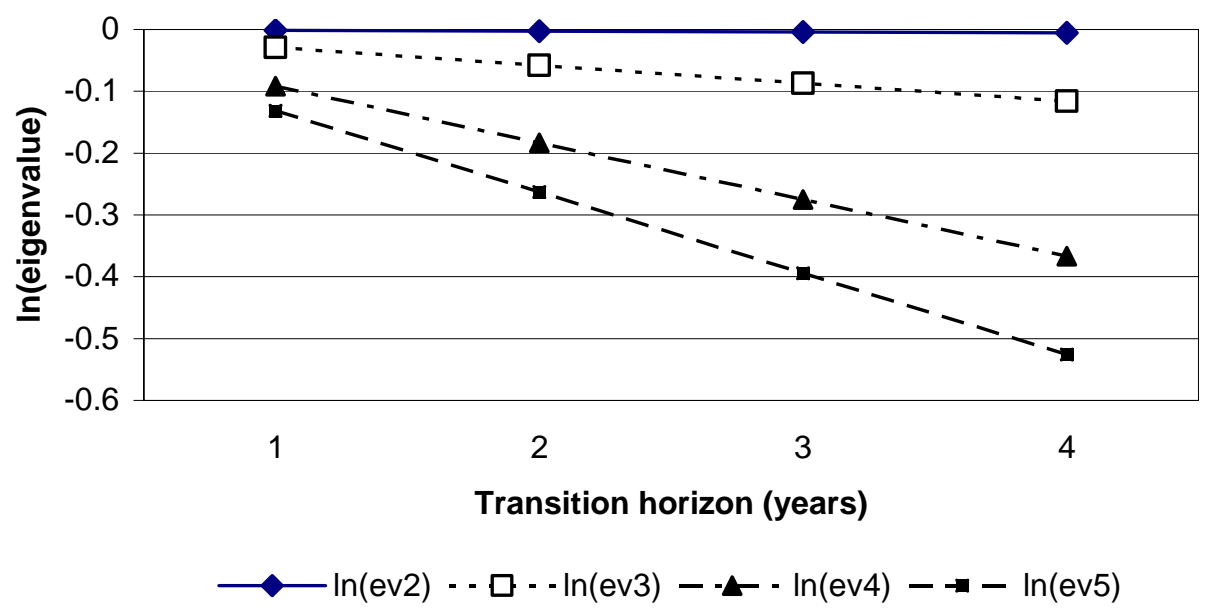

Panel B: Second Eigenvector versus Transition Horizon

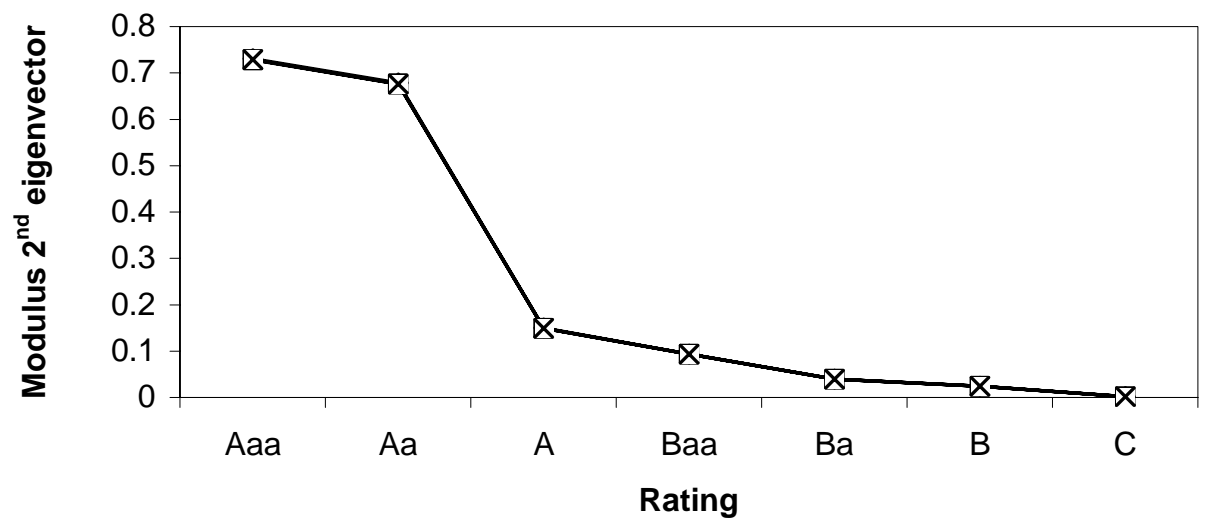

$\neg 1 Y \rightarrow \square-2 Y \triangle 3 Y-X-4 Y$

Figure 6. Spectral Decomposition of Transition Probability Matrix

Panel A reports the second to fifth largest eigenvalues of the historical transition probabilitiy matrix for horizons 1-4 years. Panel B reports the second eigenvector for horizons 1-4 years. 
TABLE 1

1-Year Historical Transition Matrix (Time-Homogeneous Estimators)

\begin{tabular}{|c|c|c|c|c|c|c|c|c|}
\hline \multicolumn{9}{|c|}{$\begin{array}{l}\text { i) Discrete multinomial (DM) estimator } \\
\text { Transition probabilities }\end{array}$} \\
\hline & Aaa & $\mathrm{Aa}$ & A & Baa & $\mathrm{Ba}$ & B & $\mathrm{C}$ & $\mathrm{D}$ \\
\hline aa & 0.944444 & 0.055556 & 0.000000 & 0.000000 & 0.000000 & 0.000000 & 0.000000 & 0.000000 \\
\hline $\mathrm{Aa}$ & 0.063954 & 0.924419 & 0.011628 & 0.000000 & 0.000000 & 0.000000 & 0.000000 & 0.000000 \\
\hline A & 0.000000 & 0.041237 & 0.886598 & 0.061856 & 0.010309 & 0.000000 & 0.000000 & 0.000000 \\
\hline Baa & 0.000000 & 0.000000 & 0.100000 & 0.854545 & 0.027273 & 0.018182 & 0.000000 & 0.033333 \\
\hline $\mathrm{Ba}$ & 0.000000 & 0.000000 & 0.000000 & 0.075758 & 0.833333 & 0.068182 & 0.015152 & 0.007576 \\
\hline B & 0.000000 & 0.000000 & 0.000000 & 0.000000 & 0.071429 & 0.845238 & 0.023810 & 0.059523 \\
\hline $\mathrm{C}$ & 0.000000 & 0.000000 & 0.000000 & 0.000000 & 0.000000 & 0.333333 & 0.500000 & 0.166667 \\
\hline $\mathrm{D}$ & 0.000000 & 0.000000 & 0.000000 & 0.000000 & 0.000000 & 0.000000 & 0.000000 & 1.000000 \\
\hline \multicolumn{9}{|c|}{$\begin{array}{l}\text { ii) Continuous homogeneous hazard rate (HHR) estimator } \\
\text { Transition intensities }\end{array}$} \\
\hline Aaa & -0.060311 & 0.060311 & 0.000000 & 0.000000 & 0.000000 & 0.000000 & 0.000000 & 0.000000 \\
\hline $\mathrm{Aa}$ & 0.063454 & -0.074991 & 0.011537 & 0.000000 & 0.000000 & 0.000000 & 0.000000 & 0.000000 \\
\hline $\mathrm{A}$ & 0.000000 & 0.038984 & -0.116953 & 0.077969 & 0.000000 & 0.000000 & 0.000000 & 0.000000 \\
\hline Baa & 0.000000 & 0.000000 & 0.100857 & -0.159690 & 0.058833 & 0.000000 & 0.000000 & 0.000000 \\
\hline $\mathrm{Ba}$ & 0.000000 & 0.000000 & 0.000000 & 0.080268 & -0.189724 & 0.102159 & 0.000000 & 0.007297 \\
\hline B & 0.000000 & 0.000000 & 0.000000 & 0.000000 & 0.080422 & -0.195310 & 0.068933 & 0.045955 \\
\hline $\mathrm{C}$ & 0.000000 & 0.000000 & 0.000000 & 0.000000 & 0.000000 & 0.302905 & -0.757261 & 0.454357 \\
\hline $\mathrm{D}$ & 0.000000 & 0.000000 & 0.000000 & 0.000000 & 0.000000 & 0.000000 & 0.000000 & 0.000000 \\
\hline \multicolumn{9}{|c|}{ Transition probabilities } \\
\hline Aaa & 0.943265 & 0.056406 & 0.000320 & 0.000320 & $1.2 \times 10^{-}$ & & $3.1 \times 10^{-11}$ & $1.9 \times 10^{-10}$ \\
\hline $\mathrm{Aa}$ & 0.059346 & 0.929742 & 0.010503 & 0.000401 & 0.000008 & $2.0 \times 10^{-7}$ & $2.3 \times 10^{-9}$ & $1.6 \times 10^{-8}$ \\
\hline $\mathrm{A}$ & 0.001138 & 0.035490 & 0.893283 & 0.068048 & 0.001967 & 0.000067 & 0.000001 & 0.000006 \\
\hline Baa & 0.000038 & 0.001751 & 0.088024 & 0.857808 & 0.049576 & 0.002529 & 0.000050 & 0.000223 \\
\hline $\mathrm{Ba}$ & $7.4 \times 10^{-7}$ & 0.000046 & 0.003472 & 0.067639 & 0.832584 & 0.085502 & 0.002450 & 0.008307 \\
\hline B & $1.2 \times 10^{-8}$ & $9.2 \times 10^{-7}$ & 0.000092 & 0.002703 & 0.066808 & 0.837991 & 0.043687 & 0.048718 \\
\hline $\mathrm{C}$ & $6.8 \times 10^{-10}$ & $5.2 \times 10^{-8}$ & $6.7 \times 10^{-6}$ & 0.000257 & 0.009356 & 0.225019 & 0.475650 & 0.289712 \\
\hline D & 0.000000 & 0.000000 & 0.000000 & 0.000000 & 0.000000 & 0.000000 & 0.000000 & 1.000000 \\
\hline
\end{tabular}

The estimates are based on Moody's ratings 1981-2004 for 72 countries. Both estimators assume time-homogeneity (over the span of the sample) in the rating migration process. 
TABle 2

Empirical Distribution of 1-year Default Probabilities (Time-Homogeneous Estimators)

\begin{tabular}{lccccccc}
\hline \hline & & \multicolumn{5}{c}{ Panel A: Discrete Multinomial (DM) estimator } \\
\cline { 3 - 8 } Rating & True $P D$ & Mean $(\widehat{P D})$ & Median $(\widehat{P D})$ & StDev $(\widehat{P D})$ & $95 \%$ & Conf. Int. & Mean Bias Median Bias \\
\hline Aaa & $1.9 \times 10^{-10}$ & 0 & 0 & 0 & {$[0,0]$} & $-1.89 \times 10^{-10}$ & $-1.89 \times 10^{-10}$ \\
Aa & $1.6 \times 10^{-8}$ & 0 & 0 & 0 & {$[0,0]$} & $-1.58 \times 10^{-8}$ & $-1.58 \times 10^{-8}$ \\
A & $5.6 \times 10^{-6}$ & 0 & 0 & 0 & {$[0,0]$} & $-5.56 \times 10^{-6}$ & $-5.56 \times 10^{-6}$ \\
Baa & 0.0002226 & 0.0002696 & 0 & 0.001502 & {$[0,0.007520]$} & 0.00004700 & -0.0002226 \\
Ba & 0.008307 & 0.008905 & 0.007519 & 0.008086 & {$[0,0.02801]$} & 0.0005980 & -0.0007883 \\
B & 0.04872 & 0.04661 & 0.04412 & 0.02596 & {$[0,0.1067]$} & -0.002110 & -0.004600 \\
C & 0.2897 & 0.2761 & 0.2500 & 0.2591 & {$[0,1]$} & -0.01360 & -0.03971 \\
\hline
\end{tabular}

Panel B: Continuous Homogeneous Hazard Rate (HHR) estimator

Rating True $P D \overline{\operatorname{Mean}(\widehat{P D})}$ Median $(\widehat{P D}) \operatorname{StDev}(\widehat{P D}) \quad 95 \%$ Conf. Int. Mean Bias Median Bias

\begin{tabular}{lccccccc}
\hline Aaa & $1.9 \times 10^{-10}$ & $2.3 \times 10^{-10}$ & $8.8 \times 10^{-11}$ & $3.5 \times 10^{-10}$ & {$\left[0,1.2 \times 10^{-9}\right]$} & $3.5 \times 10^{-11}$ & $-1.01 \times 10^{-11}$ \\
Aa & $1.6 \times 10^{-8}$ & $1.8 \times 10^{-8}$ & $7.8 \times 10^{-9}$ & $2.7 \times 10^{-8}$ & {$\left[0,9 \times 10^{-8}\right]$} & $2.6 \times 10^{-9}$ & $-7.99 \times 10^{-9}$ \\
A & $5.6 \times 10^{-6}$ & $6.6 \times 10^{-6}$ & $5.1 \times 10^{-6}$ & $6.8 \times 10^{-6}$ & {$\left[1.8 \times 10^{-7}, 2.4 \times 10^{-5}\right]$} & $9.9 \times 10^{-7}$ & $-4.91 \times 10^{-7}$ \\
Baa & 0.0002226 & 0.0002701 & 0.0002150 & 0.0002625 & {$\left[1.1 \times 10^{-5}, 0.0009718\right]$} & 0.00004748 & $-7.59 \times 10^{-6}$ \\
Ba & 0.008307 & 0.01003 & 0.009177 & 0.007823 & {$[0.0007534,0.02963]$} & 0.001727 & 0.0008696 \\
B & 0.04872 & 0.05861 & 0.05364 & 0.04568 & {$[0.01333,0.1204]$} & 0.009890 & 0.004923 \\
C & 0.2897 & 0.3220 & 0.3078 & 0.1882 & {$[0,0.7488]$} & 0.03231 & 0.01808 \\
\hline \hline
\end{tabular}

True $P D$ refers to the historical probabilities of default, signified in bold in Table 1, adopted as parameter values in

the ratings DGP for the bootstrap simulations. The bias is defined as the estimated value minus the true value. 
TABLE 3

Empirical Distribution of Continuous versus Discrete Estimator

\begin{tabular}{lcccc}
\hline \hline & \multicolumn{3}{c}{ Panel A: 1-year default probability } \\
\cline { 2 - 5 } Rating & Mean $(\Delta \widehat{P D})$ & $\operatorname{StDev}(\Delta \widehat{P D})$ & $95 \%$ Conf. Int. & $\begin{array}{c}\text { Bootstrap test } \\
\mathrm{H}_{0}: \Delta P D=0\end{array}$ \\
\hline Aaa & $2.3 \times 10^{-10}$ & $3.5 \times 10^{-10}$ & {$\left[0,1.2 \times 10^{-9}\right]$} & Not reject \\
Aa & $1.8 \times 10^{-8}$ & $2.7 \times 10^{-8}$ & {$\left[0,9 \times 10^{-8}\right]$} & Not reject \\
A & $6.6 \times 10^{-6}$ & $6.8 \times 10^{-6}$ & {$\left[1.8 \times 10^{-7}, 2.4 \times 10^{-5}\right]$} & Reject \\
Baa & $4.8 \times 10^{-7}$ & 0.001486 & {$[-0.006857,0.000946]$} & Not reject \\
Ba & 0.001129 & 0.004858 & {$[-0.01078,0.01024]$} & Not reject \\
B & 0.01200 & 0.03948 & {$[-0.01468,0.04364]$} & Not reject \\
C & 0.04557 & 0.2171 & {$[-0.3796,0.5269]$} & Not reject \\
\hline
\end{tabular}

Panel B: Overall migration risk

\begin{tabular}{|c|c|c|c|c|c|}
\hline$\hat{m}$ & $\Delta \hat{m}$ & $\operatorname{Mean}(\Delta \hat{m})$ & $\operatorname{StDev}(\Delta \hat{m})$ & 95\% Conf. Int. & $\mathrm{H}_{0}: \Delta m=0$ \\
\hline $\begin{array}{c}0.1921(\mathrm{HHR}) \\
0.1954(\mathrm{DM})\end{array}$ & -0.003257 & -0.09424 & 0.04160 & {$[-0.1801,-0.03485]$} & Reject \\
\hline
\end{tabular}


TABLE 4

2-Year and 3-Year Historical Transition Probabilities (Homogeneous Estimators)

\begin{tabular}{lcccccccc}
\hline \multicolumn{7}{c}{ Panel A: 2-year transition horizon } \\
\multicolumn{7}{c}{ Discrete multinomial } & $(\mathrm{DM})$ estimator \\
Aaa & 0.893939 & 0.106061 & 0.000000 & 0.000000 & 0.000000 & 0.000000 & 0.000000 & 0.000000 \\
Aa & 0.132530 & 0.843373 & 0.024096 & 0.000000 & 0.000000 & 0.000000 & 0.000000 & 0.000000 \\
A & 0.000000 & 0.093023 & 0.837209 & 0.046512 & 0.023256 & 0.000000 & 0.000000 & 0.000000 \\
Baa & 0.000000 & 0.000000 & 0.192308 & 0.730769 & 0.038462 & 0.019230 & 0.000000 & 0.019231 \\
Ba & 0.000000 & 0.000000 & 0.015873 & 0.126984 & 0.730159 & 0.111111 & 0.015873 & 0.000000 \\
B & 0.000000 & 0.000000 & 0.000000 & 0.025641 & 0.128205 & 0.717949 & 0.025641 & 0.102564 \\
C & 0.000000 & 0.000000 & 0.000000 & 0.000000 & 0.000000 & 1.000000 & 0.000000 & 0.000000 \\
D & 0.000000 & 0.000000 & 0.000000 & 0.000000 & 0.000000 & 0.000000 & 0.000000 & 1.000000 \\
& & Continuous homogeneous hazard rate & $($ HHR $)$ estimator & \\
Aaa & 0.893097 & 0.105661 & 0.001181 & 0.000059 & 0.000002 & $6.4 \times 10^{-8}$ & $2.0 \times 10^{-9}$ & $5.9 \times 10^{-9}$ \\
Aa & 0.111168 & 0.868141 & 0.019202 & 0.001432 & 0.000054 & 0.000003 & $5.0 \times 10^{-8}$ & $2.5 \times 10^{-7}$ \\
A & 0.004199 & 0.064883 & 0.804324 & 0.119306 & 0.006774 & 0.000456 & 0.000013 & 0.000044 \\
Baa & 0.000272 & 0.006259 & 0.154329 & 0.745187 & 0.084146 & 0.008588 & 0.000293 & 0.000927 \\
Ba & 0.000011 & 0.000323 & 0.011955 & 0.114803 & 0.702356 & 0.144981 & 0.007061 & 0.018510 \\
B & $3.2 \times 10^{-7}$ & 0.000013 & 0.000629 & 0.009133 & 0.112473 & 0.726370 & 0.057355 & 0.094027 \\
C & $4.3 \times 10^{-8}$ & 0.000001 & 0.000089 & 0.001621 & 0.029437 & 0.345947 & 0.241067 & 0.381837 \\
D & 0.000000 & 0.000000 & 0.000000 & 0.000000 & 0.000000 & 0.000000 & 0.000000 & 1.000000
\end{tabular}

Panel B: 3-year transition horizon

Discrete multinomial (DM) estimator

$\begin{array}{lllllllll}\text { Aaa } & 0.825000 & 0.175000 & 0.000000 & 0.000000 & 0.000000 & 0.000000 & 0.000000 & 0.000000 \\ \text { Aa } & 0.200000 & 0.763636 & 0.036364 & 0.000000 & 0.000000 & 0.000000 & 0.000000 & 0.000000 \\ \text { A } & 0.000000 & 0.153846 & 0.730769 & 0.076923 & 0.038462 & 0.000000 & 0.000000 & 0.000000 \\ \text { Baa } & 0.000000 & 0.000000 & 0.233333 & 0.633333 & 0.066667 & 0.033333 & 0.000000 & 0.033333 \\ \text { Ba } & 0.000000 & 0.000000 & 0.050000 & 0.150000 & 0.6000000 & 0.175000 & 0.025000 & 0.000000 \\ \text { B } & 0.000000 & 0.000000 & 0.000000 & 0.052632 & 0.105263 & 0.789474 & 0.052632 & 0.000000 \\ \text { C } & 0.000000 & 0.000000 & 0.000000 & 0.000000 & 1.000000 & 0.000000 & 0.000000 & 0.000000 \\ \text { D } & 0.000000 & 0.000000 & 0.000000 & 0.000000 & 0.000000 & 0.000000 & 0.000000 & 1.000000\end{array}$

Continuous homogeneous hazard rate (HHR) estimator

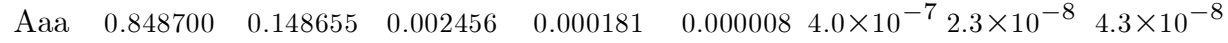

$\begin{array}{lllllllll}\text { Aa } & 0.156403 & 0.814102 & 0.026432 & 0.002888 & 0.000160 & 0.000012 & 2.0 \times 10^{-7} & 0.000001\end{array}$

$\begin{array}{llllllllll}\text { A } & 0.008732 & 0.089315 & 0.729704 & 0.157549 & 0.013178 & 0.001316 & 0.000062 & 0.000143\end{array}$

$\begin{array}{lllllllll}\text { Baa } & 0.000831 & 0.012623 & 0.203798 & 0.655469 & 0.107861 & 0.016567 & 0.000653 & 0.002197\end{array}$

$\begin{array}{llllllllll}\mathrm{Ba} & 0.000048 & 0.000955 & 0.023257 & 0.147158 & 0.600462 & 0.186280 & 0.012676 & 0.029163\end{array}$

$\begin{array}{llllllllll}\text { B } & 0.000002 & 0.000058 & 0.001808 & 0.017542 & 0.143519 & 0.649375 & 0.052951 & 0.134745\end{array}$

$\begin{array}{llllllllll}\text { C } & 4.9 \times 10^{-7} & 0.000006 & 0.000402 & 0.004196 & 0.054507 & 0.399945 & 0.186006 & 0.354937\end{array}$

$\begin{array}{llllllllll}\mathrm{D} & 0.000000 & 0.000000 & 0.000000 & 0.000000 & 0.000000 & 0.000000 & 0.000000 & 1.000000\end{array}$

Panel $C$ : Overall migration risk for increasing time horizon

\begin{tabular}{cccc} 
Horizon (years) & $\hat{m}($ HHR $)$ & $\hat{m}(\mathrm{DM})$ & $\Delta \hat{m}($ HHR-DM $)$ \\
\hline 1 & 0.1921 & 0.1954 & -0.0033 \\
2 & 0.3155 & 0.3916 & -0.0761 \\
3 & 0.3802 & 0.4661 & -0.0859 \\
\hline \hline
\end{tabular}


TABLE 5

2-Year and 3-Year Historical Transition Probabilities (Continuous Estimators)

\begin{tabular}{|c|c|c|c|c|c|c|c|c|}
\hline \multicolumn{9}{|c|}{ Panel A: 2-year transition horizon } \\
\hline \multicolumn{9}{|c|}{ Non-homogeneous hazard rate (NHHR) estimator } \\
\hline & Aaa & $\mathrm{Aa}$ & A & Baa & $\mathrm{Ba}$ & $\mathrm{B}$ & $\mathrm{C}$ & $\mathrm{D}$ \\
\hline Aaa & 0.920163 & 0.079837 & 0.000000 & 0.000000 & 0.000000 & 0.000000 & 0.000000 & 0.000000 \\
\hline a & 0.171438 & 0.803105 & 0.025457 & 0.000000 & 0.000000 & 0.000000 & 0.000000 & 0.000000 \\
\hline A & 0.003365 & 0.061066 & 0.786007 & 0.138133 & 0.010760 & 0.000670 & & 0.000000 \\
\hline Baa & 0.000000 & 0.001871 & 0.156406 & 0.717529 & 0.119186 & 0.004890 & 0.000059 & 0.000059 \\
\hline $3 a$ & 0.000000 & 0.000068 & 0.013681 & 0.103379 & 159 & 0.130877 & 0.0 & 0.019082 \\
\hline$B$ & 0.000000 & 0.00 & 0.000160 & 0.005250 & 0.111325 & 0.77 & 0.02 & 597 \\
\hline $\mathrm{C}$ & 0.000000 & 0.0 & 0.00 & 0.00 & 00 & 0.1 & 0.6 & 254 \\
\hline . & 0.000000 & 0.000000 & 0.000000 & 0.000000 & 0.000000 & 0.000000 & & 1.000000 \\
\hline \multicolumn{9}{|c|}{ Homogeneous hazard rate (HHR) estimator } \\
\hline & 0.959259 & 0.040741 & 0.000000 & 0.000000 & 0.000000 & 0.000000 & 0.000000 & 0.000000 \\
\hline $\mathrm{Aa}$ & 0.096440 & 0.887919 & 0.015641 & 0.000 & 0.0 & 0.00 & 0.00 & 0.000000 \\
\hline $\mathrm{A}$ & 0.001886 & 0.029711 & 0.887680 & 0.078201 & 0.002398 & 0.000112 & 0.000004 & 0.000007 \\
\hline $\mathrm{Baa}$ & 0.000121 & 0.000790 & 0.081537 & 0.858642 & 0.056745 & 0.001957 & 0.000065 & 0.000144 \\
\hline $\mathrm{Ba}$ & 0.000004 & 0.0000 & 0.003377 & 0.061053 & 0.853624 & 0.070544 & 0.002444 & 0.008924 \\
\hline B & 0.000001 & $4.6 \times 10^{-8}$ & 0.000158 & 0.003381 & 0.056722 & 0.867210 & 0.032466 & 0.040061 \\
\hline 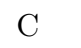 & 0.000000 & 0.000000 & 0.000003 & 0.000240 & 0.004266 & 0.078548 & 0.789233 & 0.127710 \\
\hline $\mathrm{D}$ & 0.000000 & 0.000000 & 0.000000 & 0.000000 & 0.000000 & 0.000000 & 0.000000 & 1.000000 \\
\hline
\end{tabular}

Panel B: 3-year transition horizon

Non-homogeneous hazard rate (NHHR) estimator

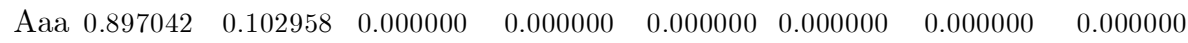

$\begin{array}{lllllllll}\mathrm{Aa} & 0.247916 & 0.715125 & 0.036959 & 0.000000 & 0.000000 & 0.000000 & 0.000000 & 0.000000\end{array}$

$\begin{array}{llllllllll}\text { Aaa } & 0.004773 & 0.085455 & 0.750384 & 0.144077 & 0.013085 & 0.001625 & 0.000021 & 0.000580\end{array}$

$\begin{array}{llllllllll}\text { Baa } & 0.000000 & 0.002713 & 0.226937 & 0.582570 & 0.176119 & 0.009498 & 0.000164 & 0.001998\end{array}$

$\begin{array}{llllllllll}\mathrm{Ba} & 0.000000 & 0.000286 & 0.027437 & 0.122774 & 0.621346 & 0.194436 & 0.007403 & 0.026319\end{array}$

$\begin{array}{llllllllll}\mathrm{B} & 0.000000 & 0.000052 & 0.004123 & 0.011141 & 0.133116 & 0.706878 & 0.039027 & 0.105663\end{array}$

$\begin{array}{llllllllll}\text { C } & 0.000000 & 0.000000 & 0.000170 & 0.002212 & 0.046899 & 0.199579 & 0.505503 & 0.245638\end{array}$

$\begin{array}{lllllllll}\mathrm{D} & 0.000000 & 0.000000 & 0.000000 & 0.000000 & 0.000000 & 0.000000 & 0.000000 & 1.000000\end{array}$

Homogeneous hazard rate (HHR) estimator

$\begin{array}{lllllllll}\text { Aaa } 0.961241 & 0.038759 & 0.000000 & 0.000000 & 0.000000 & 0.000000 & 0.000000 & 0.000000\end{array}$

$\begin{array}{llllllll}\text { Aa } & 0.113318 & 0.868130 & 0.018179 & 0.000365 & 0.000007 & 6.7 \times 10^{-8} 8.8 \times 10^{-10} 8.8 \times 10^{-10}\end{array}$

$\begin{array}{llllllllll}\text { A } & 0.002393 & 0.037180 & 0.873991 & 0.083385 & 0.002881 & 0.000159 & 0.000007 & 0.000005\end{array}$

$\begin{array}{lllllllll}\text { Baa } & 0.000157 & 0.001286 & 0.085887 & 0.842212 & 0.068326 & 0.001951 & 0.000101 & 0.000080\end{array}$

$\begin{array}{llllllllll}\mathrm{Ba} & 0.000004 & 0.000049 & 0.003032 & 0.049545 & 0.857125 & 0.083717 & 0.003419 & 0.003109\end{array}$

$\begin{array}{llllllllll}\text { B } & 1.2 \times 10^{-7} & 0.000002 & 0.000131 & 0.001896 & 0.034591 & 0.869301 & 0.041519 & 0.052561\end{array}$

$\begin{array}{lllllllll}\text { C } & 2.1 \times 10^{-8} & 1.9 \times 10^{-7} & 0.000021 & 0.000362 & 0.009194 & 0.141598 & 0.620984 & 0.227840\end{array}$

$\begin{array}{lllllllll}\mathrm{D} & 0.000000 & 0.000000 & 0.000000 & 0.000000 & 0.000000 & 0.000000 & 0.000000 & 1.000000\end{array}$

Panel $C$ : Overall migration risk for increasing time horizon

\begin{tabular}{cccc} 
Horizon (years) & $\hat{m}($ HHR $)$ & $\hat{m}($ NHHR $)$ & $\Delta \hat{m}($ NHHR-HHR $)$ \\
\hline 1 & 0.1219 & 0.1303 & 0.0084 \\
2 & 0.1352 & 0.2399 & 0.1047 \\
3 & 0.1718 & 0.3146 & 0.1428 \\
\hline \hline
\end{tabular}


TABLE 6

Empirical Distribution of 2-year Default Probabilities (Continuous Estimators)

Panel A: Homogeneous Hazard Rate (HHR) estimator

Rating True $P D \quad$ Mean $(\widehat{P D})$ StDev $(\widehat{P D})$ Median $(\widehat{P D}) \quad 95 \%$ Conf. Int. Mean Bias Median Bias

\begin{tabular}{lccccccc}
\hline Aaa & 0 & 0 & 0 & 0 & 0 & 0 & 0 \\
Aa & 0 & 0 & 0 & 0 & 0 & 0 & 0 \\
A & $4.8 \times 10^{-6}$ & $8.5 \times 10^{-6}$ & $1.3 \times 10^{-5}$ & $4.3 \times 10^{-6}$ & {$\left[0,4.3 \times 10^{-5}\right]$} & $3.7 \times 10^{-6}$ & $-4.9 \times 10^{-7}$ \\
Baa & $1.2 \times 10^{-4}$ & 0.0001625 & 0.0001979 & 0.00009753 & {$[0,0.0006900]$} & $4.2 \times 10^{-5}$ & $-2.3 \times 10^{-5}$ \\
Ba & 0.02519 & 0.009670 & 0.007105 & 0.008813 & {$[0.0006235,0.02601]$} & -0.01552 & -0.01638 \\
B & 0.09319 & 0.04327 & 0.01940 & 0.04068 & {$[0.01313,0.08646]$} & -0.04992 & -0.05251 \\
C & 0.2232 & 0.1095 & 0.06122 & 0.1079 & {$[0,0.2437]$} & -0.1137 & -0.1154 \\
\hline
\end{tabular}

Panel B: Non-Homogeneous Hazard Rate (NHHR) Estimator

Rating True $P D$ Mean $(\widehat{P D})$ StDev $(\widehat{P D})$ Median $(\widehat{P D}) \quad 95 \%$ Conf. Int. Mean Bias Median Bias

\begin{tabular}{lccccccc}
\hline Aaa & 0 & 0 & 0 & 0 & {$[0,0]$} & 0 & 0 \\
Aa & 0 & 0 & 0 & 0 & {$[0,0]$} & 0 & 0 \\
A & $4.8 \times 10^{-6}$ & $3.7 \times 10^{-6}$ & $1.7 \times 10^{-5}$ & 0 & {$\left[0,4.5 \times 10^{-5}\right]$} & $-1.1 \times 10^{-6}$ & $-4.8 \times 10^{-6}$ \\
Baa & $1.2 \times 10^{-4}$ & 0.0001218 & 0.0002324 & 0 & {$[0,0.0007751]$} & $1.7 \times 10^{-6}$ & -0.0001202 \\
Ba & 0.02519 & 0.02390 & 0.01384 & 0.02305 & {$[0.002650,0.05420]$} & -0.001292 & -0.002139 \\
B & 0.09319 & 0.09017 & 0.03512 & 0.08712 & {$[0.02992,0.1674]$} & -0.003022 & -0.006063 \\
$\mathrm{C}$ & 0.2232 & 0.1690 & 0.09032 & 0.1673 & {$[0,0.3522]$} & -0.05425 & -0.05589 \\
\hline \hline
\end{tabular}

True $P D$ refers to the default probabilities (sample estimates) adopted as parameter values in the time-heterogeneous bootstrap DGP. The bias is defined as the estimated value minus the true value. 
TABLE 7

Empirical Distribution of Homogeneous versus Heterogeneous Estimator

\begin{tabular}{lcccc}
\hline \hline & \multicolumn{4}{c}{ Panel A: 2-year default probability } \\
\cline { 2 - 5 } Rating & $\operatorname{Mean}(\Delta \widehat{P D})$ & $\operatorname{StDev}(\Delta \widehat{P D})$ & $95 \%$ Conf. Int. & $\begin{array}{c}\text { Bootstrap Test } \\
\mathrm{H}_{0}: \Delta P D=0\end{array}$ \\
\hline Aaa & 0 & 0 & 0 & - \\
Aa & 0 & 0 & 0 & - \\
A & $-4.8 \times 10^{-6}$ & $2 \times 10^{-5}$ & {$\left[-4 \times 10^{-5}, 3.5 \times 10^{-5}\right]$} & Not reject \\
Baa & $-4.1 \times 10^{-5}$ & 0.0002902 & {$[-0.0006211,0.0006058]$} & Not reject \\
Ba & 0.01423 & 0.009264 & {$[-0.0002048,0.03332]$} & Not reject \\
B & 0.04689 & 0.02326 & {$[0.007110,0.09616]$} & Reject \\
C & 0.05947 & 0.06409 & {$[-0.04547,0.2122]$} & Notreject \\
\hline
\end{tabular}

Panel B: Overall migration risk

\begin{tabular}{|c|c|c|c|c|c|}
\hline$\hat{m}$ & $\Delta \hat{m}$ & $\operatorname{Mean}(\Delta \hat{m})$ & $\operatorname{StDev}(\Delta \hat{m})$ & 95\% Conf. Int. & $\mathrm{H}_{0}: \Delta m=0$ \\
\hline $\begin{array}{c}0.2399(\mathrm{NHHR}) \\
0.1352(\mathrm{HHR})\end{array}$ & 0.1047 & 0.08104 & 0.01623 & {$[0.05331,0.1201]$} & Reject \\
\hline
\end{tabular}

$\overline{\triangle \triangle P D \text { is the non-homogeneous hazard rate (NHHR) default probability minus the homo- }}$ geneous hazard rate (HHR) default probability. Likewise for the mobility differential $\Delta m$. 
TABLE 8

Logit Estimates and Tests for Non-Markov Effects

\begin{tabular}{|c|c|c|c|c|c|}
\hline \multicolumn{6}{|c|}{ Panel A: Duration effect } \\
\hline \multirow[b]{2}{*}{ Coefficient } & \multirow[b]{2}{*}{ Effect } & \multicolumn{2}{|c|}{ Upgrade } & \multicolumn{2}{|c|}{ Downgrade } \\
\hline & & Estimate & $t$ ratio & Estimate & $t$ ratio \\
\hline$\beta$ & duration & -1.88 & -5.33 & -1.66 & -5.74 \\
\hline$\gamma_{1}$ & 1998 & 1.99 & 2.71 & -0.31 & -0.37 \\
\hline$\gamma_{1}$ & 1999 & 0.48 & 0.39 & 1.68 & 3.12 \\
\hline$\gamma_{2}$ & 2000 & 1.36 & 1.69 & 1.49 & 2.62 \\
\hline$\gamma_{3}$ & 2001 & 3.81 & 4.88 & 0.37 & 0.43 \\
\hline$\gamma_{4}$ & 2002 & 2.39 & 2.89 & 1.94 & 2.70 \\
\hline$\gamma_{5}$ & 2003 & 3.78 & 5.67 & -0.08 & -0.12 \\
\hline$\gamma_{6}$ & 2004 & -0.44 & -0.67 & -0.90 & -1.66 \\
\hline$\alpha$ & constant & -1.57 & -1.50 & -0.41 & -1.15 \\
\hline$\xi$ & heterosk. & 0.27 & 1.04 & 0.11 & 0.44 \\
\hline
\end{tabular}

Panel B: Momentum effect

\begin{tabular}{ccccccc}
\hline & & \multicolumn{2}{c}{ Upgrade } & & \multicolumn{2}{c}{ Downgrade } \\
\cline { 7 - 7 } \cline { 6 - 7 } Coefficient & Effect & Estimate & $t$ ratio & & Estimate & $t$ ratio \\
\cline { 6 - 7 }$\beta$ & rating drift & -1.24 & -1.02 & & 1.20 & 2.94 \\
$\gamma_{1}$ & 1998 & 1.65 & 2.75 & & -0.37 & -0.46 \\
$\gamma_{2}$ & 1999 & 0.60 & 0.71 & & 1.21 & 2.67 \\
$\gamma_{3}$ & 2000 & 0.84 & 1.19 & & 0.71 & 1.47 \\
$\gamma_{4}$ & 2001 & 1.83 & 3.23 & & -0.79 & -0.99 \\
$\gamma_{5}$ & 2002 & 0.84 & 1.16 & & 0.35 & 0.63 \\
$\gamma_{6}$ & 2003 & 2.61 & 5.47 & & -0.28 & -0.45 \\
$\gamma_{7}$ & 2004 & 0.90 & 1.08 & & -0.08 & -0.12 \\
$\alpha$ & constant & -6.37 & -14.46 & & -5.18 & -14.06 \\
$\xi$ & heterosk. & -0.027 & -0.35 & & -0.17 & -2.10 \\
\hline \hline
\end{tabular}

The table reports maximum likelihood estimates based on monthly ratings 1981m3-2004m3. The logit models in Panel A (B) represent the probability of rating migration as a function of duration (rating drift). $\gamma_{j}, j=1, \ldots, 7$ are year dummies and $\alpha$ is the intercept. A positive (negative) $\xi$ indicates that the error variance is higher (lower) for industrialized countries. 